\title{
Small Gd(III) Tags for Gd(III)-Gd(III) Distance Measurements in Proteins by EPR Spectroscopy
}

G. Prokopiou ${ }^{1}$, M. D. Lee ${ }^{2}$, A. Collauto ${ }^{1}$, E. H. Abdelkader ${ }^{3}$, T. Bahrenberg ${ }^{1}$, A. Feintuch ${ }^{1}$, M. Ramirez-Cohen ${ }^{1}$, J. Clayton ${ }^{4}$, J. D. Swarbrick², B. Graham²*, G. Otting ${ }^{3 *}$, D. Goldfarb ${ }^{1 *}$

${ }^{1}$ Department of Chemical Physics, Weizmann Institute of Science, Rehovot, 76100, Israel

${ }^{2}$ Monash Institute of Pharmaceutical Sciences, Monash University, Parkville, VIC 3052, Australia

${ }^{3}$ Research School of Chemistry, Australian National University, Canberra, ACT 2601 Australia

${ }^{4}$ Department of Physics, University of California, Santa-Barbara, CA CA 93106-9530, USA

Corresponding authors: B. Graham (bimbazompa@gmail.com), G. Otting (gottfried.otting@anu.edu.au), D. Goldfarb (Daniella.goldfarb@weizmann.ac.il)

Key words : Distance measurements, DEER, Gd(III) tags, spin labeling, ENDOR, high field EPR, zero-field splitting.

\section{Abstract}

The C7-Gd and C8-Gd tags are compact hydrophilic cyclen-based lanthanide tags for conjugation to cysteine residues in proteins. The tags are enantiomers, which differ in the configuration of the 2-hydroxylpropyl pendant arms coordinating the lanthanide ion. Here, we report the electron paramagnetic resonance (EPR) performance of the C7-Gd ( $S$ configuration) and C8-Gd ( $R$ configuration) tags loaded with $\mathrm{Gd}(\mathrm{III})$ on two mutants of the homodimeric ERp29 protein. The W-band EPR spectra were found to differ between the tags in the free state and after conjugation to the protein. In addition, the spectra were sensitive to the labeling position, which may originate from an environment-dependent charge density on the $\mathrm{Gd}(\mathrm{III})$-coordinating oxygens. This is in agreement with previous NMR experiments with different lanthanide ions, which suggested sensitivity to $\mathrm{H}$ bonding. W-band ${ }^{1} \mathrm{H}$-ENDOR (electron-electron double resonance) experiments detected effects from orientation selection in the central transition, due to a relatively narrow distribution in the ZFS parameters as indicated by simulations. In contrast, the distance distributions derived from DEER (double electron-electron resonance) measurements were insensitive to the $R$ or $S$ configuration of the tags and did not exhibit any orientation selection effects. The DEER measurements faithfully reflected the different widths of the distance distributions at the different protein sites in agreement with previous DEER measurements using other Gd(III) tags. Due to their small size, short tether to the protein, and a broad central EPR transition, the C7-Gd and C8-Gd tags are attractive Gd(III) tags for measurements of relatively short ( $<4 \mathrm{~nm})$ distances by EPR spectroscopy. 


\section{Introduction}

In the last decade, distance measurements by pulse dipolar electron-spin resonance spectroscopy, particularly DEER (electron-electron double resonance), have become a well-established tool for structural studies of proteins in solution..$^{1-2}$ Distance distributions in the 1.6-8 $\mathrm{nm}$ range (and up to $16 \mathrm{~nm}$ for deuterated proteins ${ }^{3}$ ) can be obtained between pairs of usually identical spin labels that are attached at two different sites of a biomacromolecule. ${ }^{2}$ Nitroxide spin labels are employed most frequently, but paramagnetic metal ions such as $\mathrm{Cu}(\mathrm{II}),{ }^{4-5} \mathrm{Mn}(\mathrm{II})^{6-8}$ and $\mathrm{Gd}(\mathrm{III})^{9-10}$ can also be used. Gd(III)-based tags (electron spin $S=7 / 2$ ) stand out for their utility at Q- ( $-34 \mathrm{GHz})$ and more so at W- ( 95 GHz) band, mostly for Gd(III)-Gd(III) $)^{11-19}$ distance measurements but also for Gd(III)-nitroxide measurements. ${ }^{20-23}$

The performance of Gd(III)-based spin labels in DEER measurements depends on the spectroscopic character of the Gd(III) ion, which in turn is a function of the ligand field created by the chelating molecule. Ideally, a Gd(III) tag for distance measurements should feature (i) a high binding affinity for the Gd(III) ion, (ii) a predictable and fixed location of the Gd(III) ion within the complex, (iii) efficient conjugation chemistry to achieve high labeling efficiency to the target protein, (iv) low flexibility of the tether connecting the tag to the protein in order to avoid broadening of the distance distributions due to tag dynamics, (v) minimal perturbation of the protein structure, (vi) a narrow EPR spectrum for maximal sensitivity, keeping in mind that, for tags with very small zero field splitting (ZFS) and Gd(III)-Gd(III) distances below $4 \mathrm{~nm}$, the data analysis becomes complicated by the presence of the pseudo-secular term of the dipolar interaction, ${ }^{24-25}$ (vii) a long phase memory time to access long distances and increase sensitivity, and (viii) sufficiently rapid spin-lattice relaxation to allow efficient data accumulation.

Site-specific Gd(III) labeling of a protein is usually achieved by ligating the tag to cysteine residues, either via S$\mathrm{S}$ bonds ${ }^{26}$ or a thioether linkage $\mathrm{e}^{26-29}$, where the latter is particularly suitable for in-cell measurements due to its stability under reducing conditions. Ligation to site-specifically introduced unnatural amino acids has also been demonstrated. ${ }^{17}$ To date, about a dozen different Gd(III) tags have been evaluated for distance measurements. All of them feature an EPR spectrum with similar characteristics, including a single, structure-less and relatively sharp line corresponding to the central $|-1 / 2\rangle \rightarrow|+1 / 2\rangle$ transition with a full-width at half-height ranging from 40 $\mathrm{MHz}$ to $300 \mathrm{MHz}$, superimposed on a rather featureless background arising from all the other transitions. ${ }^{9}$ The width of this background, just like that of the central transition, varies from tag to tag. The featureless shape of these spectra is characteristic of Gd(III) complexes in frozen solutions. It arises from large distributions of the ZFS parameters $D$ and $E$, with $E / D$ ratios reaching the maximum value of $1 / 3 .{ }^{30}$ While the center of the distribution of $D$ and $E$ values varies from tag to tag, the overall shape of the distribution is generally the same, resulting in vanishing orientation dependence in DEER measurements ${ }^{31}$ and in high tolerance for effects from the dipolar pseudo-secular terms in the spin Hamiltonian. ${ }^{24-25}$ This makes DEER measurements with Gd(III) tags uniquely attractive at high magnetic field strengths. To an important extent, however, the effectiveness of the DEER 
experiment is determined by the line-shape of the EPR signal, which in turn is governed by the ZFS. Small ZFS values ( $D \sim 500-700 \mathrm{MHz}$ ), as found for the $\mathrm{Gd}(\mathrm{III})$ complexes of the $\mathbf{C 1}^{16}$, maleimide-DO3A ${ }^{27}$, MTS-ADO3A ${ }^{13}$ and $\mathbf{C 9}^{12}$ tags (see Fig. 1 and Fig. S1 in the supplementary information (SI) for chemical structures), are excellent for long distances ( $>4 \mathrm{~nm}$ ) but less suited for short distances, where the weak coupling approximation assumed in standard data analysis leads to artificial broadening of the distance distribution. ${ }^{24,32-33}$ This broadening for short distances can be overcome with tags featuring $D$ values $>1100 \mathrm{MHz}$, which leads to loss in sensitivity, or by using a large frequency separation between the pump and observe pulse. The latter requires non-standard instrumentation. ${ }^{32}$ Alternatively, RIDME experiments do not suffer from artificial broadening of distance distributions for short distances, but come with other complications. ${ }^{33-34}$

In contrast to nitroxide spin labels, which are sensitive to the proticity and polarity of their immediate environment, ${ }^{35}$ and therefore convey information about properties such as localization in membranes versus solvent exposure, ${ }^{36}$ the EPR spectra of all Gd(III) tags studied to date have shown no clear dependence on the conjugation site on the protein. Nonetheless, although the EPR spectra per se are insensitive to local properties of the protein such as polarity or charge distribution, assessment of local water content in the vicinity of Gd(III) spin labels is possible by ${ }^{2} \mathrm{H}$ ESEEM (electron-spin echo envelope modulation) and ENDOR experiments ${ }^{19}$ as has also been shown for nitroxides. ${ }^{37-38}$

In this work, we evaluate two enantiomers of a new Gd(III) tag for DEER applications, which feature an EPR spectrum that is sensitive to its local environment, and compare their performance with previously published Gd(III) tags. For simplicity, as all tags were prepared with Gd(III), we refer to them in the following by their name without explicitly stating the presence of a Gd(III) ion. The tags are compact in size, feature a short linker and are chirally pure, with $S$ and $R$ configurations of the 2-hydroxypropyl pendant arms in the C7 and C8 tag, respectively (Fig. 1). ${ }^{39}$ Compared to the bulky phenylethylacetamide arms present in the $\mathbf{C 1}^{16}$ and $\mathbf{C} \mathbf{9}^{12}$ tags (Fig. 1), shown to restrict tag movements by NMR, ${ }^{26,40-41}$ the 2-hydroxypropyl pendant arms of the $\mathbf{C 7}$ and $\mathbf{C 8}$ tags are much smaller and more hydrophilic. Despite their small size, the $\mathbf{C 7}$ and $\mathbf{C 8}$ tags restrict tag movements quite well by virtue of a short tether between the protein and the paramagnetic metal ion, as indicated by large pseudocontact shifts observed in their NMR spectra. ${ }^{39}$ This suggests that these tags can yield narrow distance distributions. Furthermore, the tags are structurally similar to the $(S)$-THP and $(R)$-THP contrast agents used in magnetic resonance imaging, which have been reported to be responsive to inner- and outer-sphere interactions of the metal with coordinating counter anions. ${ }^{42}$

We tested the performance of the $\mathbf{C 7}$ and $\mathbf{C 8}$ tags on the rat ERp29 protein. ERp29 is a homodimer ${ }^{16,43-44}$ and therefore only a single labeling site is required for DEER measurements. Consequently, the EPR spectrum represents the properties of a unique site. As the W-band EPR spectra showed unusual line-shapes and sensitivity with respect to the labeling position, we carried out EPR spectral simulations and detailed ${ }^{1} \mathrm{H}$ ENDOR studies to understand the spectral differences between free and protein-bound tags. The ${ }^{1} \mathrm{H}$ ENDOR spectrum was only 
slightly sensitive towards different tag environments or tag chiralities, but it showed clear effects from orientation selection, as different orientations of the tag with respect to the magnetic field could be selected at varied magnetic field settings. In contrast, our DEER results were insensitive to the tag chiralities and showed no orientation selection effects. These are desired properties for a Gd(III) tag for distance measurements. In addition, once the sensitivity of the EPR spectra of these tags to the environment is fully understood, they may be also used as reporters of local properties of the protein near the labeling site.

Figure 1. Chemical structures of Gd(III) complexes referred to in the text. (R) THP, C8, C2 and T2 are the enantiomers of (S) THP, C7, C1 and T1, respectively. In all structures, Gd indicates a Gd(III) ion.

\section{Experimental}

\section{Synthesis and materials}

Tag synthesis. The $\mathbf{C 7 / 8}$ tags were synthesized as described previously. ${ }^{39}$

Protein synthesis. The mutants S114C/C157S and G147C/C157S of ERp29 were cloned into the pETMCSIII vector $^{45}$ with an N-terminal His6 tag and produced by cell-free synthesis following an established protocol. ${ }^{46,47}$ The cell-free reactions were conducted at $30{ }^{\circ} \mathrm{C}$ for $16 \mathrm{~h}$ in a dialysis system with $2 \mathrm{~mL}$ inner reaction mixture and $20 \mathrm{~mL}$ outer buffer. For simplicity, these double mutants are referred to in the following as S114C and G147C, respectively. 
Protein purification. For protein purification a $1 \mathrm{~mL}$ Ni-NTA column (GE Healthcare, USA) was used according to the manufacturer's protocol. The purified proteins were dialyzed against $50 \mathrm{mM}$ sodium phosphate buffer (pH 8) at $4{ }^{\circ} \mathrm{C}$. Finally, the samples were concentrated using an Amicon ultrafiltration centrifugal tube with a molecular weight cutoff (MWCO) of $10 \mathrm{kDa}$. The average yield was about $1 \mathrm{mg}$ of purified protein per mL cellfree reaction mixture (inner buffer).

Protein ligation with C7 or C8. $0.1 \mathrm{mM}$ protein solution in $50 \mathrm{mM}$ sodium phosphate buffer, $\mathrm{pH}$ 8, was reduced by incubation with $5 \mathrm{mM}$ dithiothreitol (DTT) for $1 \mathrm{~h}$. Subsequently, the DTT was washed out by ultrafiltration (MWCO $10 \mathrm{kDa}$ ). The reduced protein solution was added slowly into a solution of 5 equivalents of $\mathbf{C 7}$ or $\mathbf{C 8}$ in the same buffer and kept at room temperature overnight.

Preparation of the EPR samples. After the labeling reaction, the protein samples were concentrated and exchanged to EPR buffer (20 mM 2-( $N$-morpholino)ethanesulfonic acid (MES) in $\mathrm{D}_{2} \mathrm{O}$, pH 4.9, or $20 \mathrm{mM}$ tris(hydroxymethyl)aminomethane (Tris)- $\mathrm{HCl}$ in $\mathrm{D}_{2} \mathrm{O}, \mathrm{pH}$ 8.0. $\mathrm{pH}$ values are uncorrected $\mathrm{pH}$ meter readings). The buffer exchange was performed by ultrafiltration (MWCO $10 \mathrm{kDa}$ ) and perdeuterated glycerol was added to a final concentration of $20 \%(\mathrm{v} / \mathrm{v})$ to reach a final protein concentration of $0.1 \mathrm{mM}$. The $\mathrm{pH}$ values were not corrected for H/D exchange.

Samples for EPR and ENDOR spectroscopy of the free C7 and C8 tags were prepared at a concentration of 200 $\mu \mathrm{M}$ in $\mathrm{D}_{2} \mathrm{O}$ :glycerol- $d_{8} 7: 3 \mathrm{v} / \mathrm{v}(\mathrm{pH}=6.7 \pm 0.2)$. For the $\mathrm{pH}$-dependent measurements, $2 \mu \mathrm{L}$ of a $1 \mathrm{mM}$ stock solution of $\mathbf{C 7}$ or $\mathbf{C 8}$ tag were diluted into $18 \mu \mathrm{L}$ of a previously prepared citrate/phosphate buffer of the desired $\mathrm{pH}(3,6,7,8$ or 9 , respectively). The exact buffer compositions for $100 \mu \mathrm{L}$ buffer are given in Table S1. After thorough mixing, glycerol was added $(10 \% \mathrm{v} / \mathrm{v})$ and the samples were loaded into a glass capillary with an outer diameter of $0.84 \mathrm{~mm}$. The sample for the $240 \mathrm{GHz}$ EPR measurements consisted of a $300 \mu \mathrm{M}$ solution of free $\mathbf{C 7}$ in $\mathrm{D}_{2} \mathrm{O} /$ glycerol-d 8 (30\%, v/v). For continuous wave (CW) and rapid passage EPR measurements, $8 \mu \mathrm{L}$ of sample was loaded into a Teflon sample cup, flash frozen in liquid nitrogen, and loaded into the $200 \mathrm{~K}$ pre-cooled cryostat and then further cooled to $5 \mathrm{~K}$.

\section{Spectroscopic measurements}

EPR measurements were performed on a home-built W-band spectrometer $(94.9 \mathrm{GHz})^{48-49}$ at $10 \mathrm{~K}$ using a repetition time of $1 \mathrm{~ms}$. Echo-detected EPR (ED-EPR) spectra were collected for all samples by recording the intensity of the spin echo generated by the a $\pi / 2-\tau-\pi-\tau-$ echo sequence, where the lengths of the $\pi / 2$ and $\pi$ pulses were set to $30 \mathrm{~ns}$ and $60 \mathrm{~ns}$, respectively, and the inter-pulse delay $\tau$ was set to $160 \mathrm{~ns}$. The magnetic field was swept at a rate of $0.27 \mathrm{mT} / \mathrm{s}$. A 2-step phase cycle $(\mathrm{x},-\mathrm{x})$ was applied to the $\pi / 2$ pulse and the receiver phase. Measurements at $240 \mathrm{GHz}$ were carried out at $5 \mathrm{~K}$ on a home-built spectrometer. ${ }^{50-52}$ For CW EPR measurements, the incident microwave power and modulation amplitude were adjusted to achieve maximum signal-to-noise ratio 
(SNR) and avoid artificial broadening of the line-shape. The modulation frequency was $20 \mathrm{kHz}$. The magnetic field was swept at a rate of $0.1 \mathrm{mT} / \mathrm{s}$ using the sweep coil, with the main coil of the magnet at $\sim 8.60 \mathrm{~T}$. Phase correction and baseline subtraction were applied to the raw CW EPR data as described previously. ${ }^{50}$ The rapid passage measurement was carried out as described earlier ${ }^{53}$ in a similar fashion to CW EPR measurements, with the exception that a higher microwave power was used to induce a passage regime. The main coil of the magnet was used for rapid passage measurements with a sweep rate of $0.1 \mathrm{~T} / \mathrm{min}$. Phase correction and baseline subtraction was applied to the data, choosing the final phase to maximize the integral of the absorption signal. ${ }^{50}$

Davies ${ }^{1} \mathrm{H}$-ENDOR spectra were collected by recording the intensity of the spin echo generated by the sequence $\pi_{\mathrm{mw}}-T-\pi_{\mathrm{RF}}-T-\pi / 2_{\mathrm{mw}}-\tau-\pi_{\mathrm{mw}}-\tau-$ acquisition while sweeping the radiofrequency over a $\pm 5 \mathrm{MHz}$ range centered around the ${ }^{1} \mathrm{H}$ nuclear Larmor frequency in steps of $10 \mathrm{kHz}$. The lengths of the $\pi / 2 \mathrm{mw}$ and $\pi_{\mathrm{mw}}$ microwave pulses were set to $100 \mathrm{~ns}$ and $200 \mathrm{~ns}$, respectively, the length of the $\pi_{\mathrm{RF}}$ radiofrequency pulse was optimized for inversion of the ${ }^{1} \mathrm{H}$ nuclear transitions (resulting in ${ }^{1} \mathrm{H}$ NMR pulse lengths between $25 \mu \mathrm{s}$ and $30 \mu$ ), the delay $T$ was set to $5 \mu$ s and the inter-pulse delay $\tau$ was set to $1 \mu \mathrm{s}$. A 4-step phase cycle $\phi_{1}=\mathrm{x},-\mathrm{x}, \mathrm{X},-\mathrm{x} ; \phi_{2}=\mathrm{x}, \mathrm{X},-\mathrm{x},-\mathrm{x}$; receiver $=\mathrm{X},-\mathrm{X},-\mathrm{X}, \mathrm{X}$ was applied, where $\phi_{1}$ and $\phi_{2}$ are the phases of the second $(\pi / 2)$ and third $(\pi)$ microwave pulses. A random scan of the radiofrequency was performed in order to reduce distortions arising from temperature drifts and nuclear transition saturation effects. ${ }^{54}$ The pulse sequence was supplemented with echotrain detection in order to increase the sensitivity. ${ }^{49}$ The experiments were collected as a sequence of echo-train profiles, one for each value of the radiofrequency, and the traces were integrated using the same parameters for the whole set of measurements to yield the final 1D spectra.

The ${ }^{1} \mathrm{H}$-ENDOR measurements of the free $\mathbf{C} \mathbf{8}$ and $\mathbf{C 7}$ tags were performed at the magnetic field position $B_{0}$ that corresponds to the maximum of the echo-detected EPR spectrum $\left(B_{0}=3404\right.$ mT). For the S114C-C8, S114C-C7, and G147C-C8 mutants, measurements were performed at the two different magnetic field positions corresponding to the two local maxima of the respective echo-detected EPR spectra (see Fig.2). Specifically, G147C-C8: 3401.3, 3408.4 mT, S114C-C7: 3402.5, 3404.5 mT, S114C-C8: 3400, 3408.2 mT. For the G147CC7 mutant, measurements were only performed at the magnetic field position that corresponds to the low-field local maximum of the echo-detected EPR spectrum $\left(B_{0}=3401 \mathrm{mT}\right)$.

Mims ${ }^{2} \mathrm{H}$-ENDOR spectra were collected for the ERp29 G147C-C8 sample by recording the intensity of the spin echo generated by the sequence $\pi / 2 \mathrm{mw}-\tau-\pi / 2 \mathrm{mw}-T-T_{\mathrm{RF}}-T-\pi / 2 \mathrm{mw}-\tau-$ acquisition while sweeping the radiofrequency over a $\pm 1.25 \mathrm{MHz}$ range centered around the ${ }^{2} \mathrm{H}$ nuclear Larmor frequency in steps of $10 \mathrm{kHz}$. The length of the $\pi / 2 \mathrm{mw}$ microwave pulses was set to $15 \mathrm{~ns}$, the length of the $T_{\mathrm{RF}}$ radiofrequency pulse was set to $50 \mu$ s, the delay $T$ was set to $5 \mu$ s, and the inter-pulse delay $\tau$ was set to $400 \mathrm{~ns}$. The length of the RF pulse did not achieve full inversion of the ${ }^{2} \mathrm{H}$ nuclear transition due to 5\% RF duty cycle limitations associated with the 
chosen scan repetition time. An 8-step phase cycle $\phi_{1}=\mathrm{x}, \mathrm{x}, \mathrm{X}, \mathrm{X},-\mathrm{x},-\mathrm{x},-\mathrm{x}-\mathrm{x} ; \phi_{2}=(\mathrm{x}, \mathrm{X},-\mathrm{x},-\mathrm{x})_{2} ; \phi_{3}=(\mathrm{x},-\mathrm{x})_{4}$; receiver $=\mathrm{x},-\mathrm{x},-\mathrm{x}, \mathrm{X},-\mathrm{X}, \mathrm{X}, \mathrm{X},-\mathrm{x}$ was applied, where $\phi_{1}, \phi_{2}$ and $\phi_{3}$ are the phases of the three $\pi / 2$ microwave pulses. A random scan of the radiofrequency was performed in order to reduce distortions arising from temperature drifts and nuclear transition saturation effects. The ${ }^{2} \mathrm{H}$-ENDOR measurements were performed at the same two magnetic field positions used for the ${ }^{1} \mathrm{H}$-ENDOR spectra.

DEER measurements were recorded at W-band at $10 \mathrm{~K}$ using the standard four-pulse DEER sequence, $(\pi / 2)_{v 1}-\tau_{1}-(\pi)_{v 1}-\left(\tau_{1}+t\right)-(\pi)_{v 2}-\left(\tau_{2}-t\right)-(\pi)_{v 1}-\tau_{2}-e c h o .{ }^{55}$ The parameters for experiments using the single-mode cavity were: a pump $\pi / 2$ pulse duration of $15 \mathrm{~ns}$, observer $\pi / 2$ and $\pi$ pulse durations of 15 and $30 \mathrm{~ns}$, respectively, and a frequency difference between the pump and observer pulses of $100 \mathrm{MHz}$, with the pump pulse set to the maximum of the Gd(III) EPR spectrum. The delay time $\tau_{1}$ was $375 \mathrm{~ns}$, the delay $t$ was incremented by $50 \mathrm{~ns}$ and $75 \mathrm{~ns}$ for the G147C and S114C mutants, respectively, and the repetition time was $800 \mu \mathrm{s}$. An 8-step phase cycle was employed to remove instrumental artifacts and to compensate for DC offset. The same experimental parameters were used for the dual-mode cavity, using $\Delta v=230 \mathrm{MHz}$, a pump pulse position corresponding to a field of $3394.6 \mathrm{mT}$ and observer pulses at $3386.6 \mathrm{mT}$ in a field-sweep measurement. $\tau_{1}$ was set to $375 \mathrm{~ns}$, the delay $t$ was incremented by $75 \mathrm{~ns}$, the repetition time was $0.8 \mathrm{~ms}$. The accumulation time for the DEER experiments was in the range of $14-20 \mathrm{~h}$. The DEER data were analyzed using the program DeerAnalysis $2015 .^{56}$ Distance distributions were obtained using Tikhonov regularization.

\section{Simulations}

EPR spectra were simulated using EasySpin ${ }^{57}$ including uncorrelated $D$ and $E$ strains. ENDOR spectra were simulated using the 'salt' function. An effective $S=1 / 2$ system was assumed and orientation selection was taken into account; this is how the ZFS affected the ENDOR spectra. Initially, only the central transition was considered. The selected orientations of the ZFS tensor with respect to the external magnetic field for a particular field, for which an ENDOR spectrum was recorded, were determined from a simulation of the central transition applying the "orisel" function of Easyspin with a pulse bandwidth of $100 \mathrm{MHz}$ to account for inhomogeneous broadening and to smooth the calculated ENDOR spectra. In principle, for each $D$ and $E$ value within the $D$ and $E$ distributions determined by the simulations an EPR spectrum should be calculated and the set of orientations selected for a particular field should be extracted. The final set of orientations would then be determined from all $D$ and $E$ values with the appropriate weights. In practice, to save computation time we did not consider the full set of $D$ and $E$ values spanned by the $D$ and $E$ strains but considered just two Gaussian distributions, the widths of which reflect the $D$ and $E$ strain. The first gives the $D$ distribution with a fixed $E$ value corresponding to the center of the $E$ distribution, whereas the second Gaussian gives the $E$ distribution with a fixed $D$ value corresponding to the center of the $D$ distribution. The minimum weights of the selected orientations was at least $10 \%$ of the maximum value. Finally, the ENDOR spectra were simulated using the full set of selected polar $\theta$ and azimuthal $\phi$ angles of the 
ZFS tensor with respect to the magnetic field. The relative orientation of the protons' hyperfine tensor with respect to the ZFS was taken as constant for the whole range of $D$ and $E$.

To improve the fit, we also considered contributions from the $|-3 / 2\rangle \rightarrow|-1 / 2\rangle$ and $|1 / 2\rangle \rightarrow|3 / 2\rangle$ transitions, considering only the ENDOR signals arising from the $\pm 3 / 2$ electron spin manifolds. This was done by assuming an $S=1 / 2$ spin system with a three-fold larger coupling than the coupling used to simulate the signals arising from the central transition ${ }^{58}$ and taking into account the orientations selected from $|-3 / 2\rangle \rightarrow|-1 / 2\rangle$ and $|1 / 2\rangle \rightarrow|3 / 2\rangle$ transitions as determined from the simulations of EPR spectrum described above. In this case a four-fold higher weight was given to the ENDOR lines arising from the $-3 / 2$ manifold as compared to those belonging to the $+3 / 2$ manifold. The relative weighting factor and the total contribution of these transitions were adjusted to fit the experimental spectra.

The suppression effect was taken into account by multiplying the spectra with the selectivity function ${ }^{59}$

$$
F\left[A(M H z) ; t_{i n v}(\mu S)\right]=\left\{\begin{array}{cc}
\frac{1.4\left(A \cdot t_{i n v}\right)}{0.7^{2}+\left(A \cdot t_{i n v}\right)^{2}} & A \leq \frac{0.7}{t_{i n v}} \\
1 & A>\frac{0.7}{t_{i n v}}
\end{array}\right.
$$

where $t_{\text {inv }}$ is the length of the microwave inversion $\pi$ pulse.

\section{Results}

The $\mathbf{C 7}$ and $\mathbf{C 8}$ tags are highly water-soluble, and mass spectra (see Fig. S2) indicated that the ligation with the single-cysteine mutants ERp29 S114C and G147C proceeded to at least 50\%. EPR samples were prepared for both the free tags (in $\mathrm{D}_{2} \mathrm{O}(\mathrm{pH}=6.7 \pm 0.2)$ and at $\mathrm{pH} 3,6,7,8$ and 9 in $\mathrm{H}_{2} \mathrm{O}$ ) and following ligation to the $\mathrm{S} 114 \mathrm{C}$ and G147C mutants. In the following we refer to the free tag samples prepared only in $\mathrm{D}_{2} \mathrm{O} /$ glycerol as $\mathbf{C 7}$ and C8. For samples of the free tags prepared at a controlled $\mathrm{pH}$, the $\mathrm{pH}$ value is explicitly specified. The $\mathrm{pH}$ values quoted in the present work all refer to room temperature, while spectra were recordedat low temperatures on frozen solutions. Notably, depending on the buffer used, the $\mathrm{pH}$ of solutions cooled below $0{ }^{\circ} \mathrm{C}$ can decrease or increase. The problem of changing $\mathrm{pH}$ at very low temperature due to temperature dependent proton dissociation equilibria is most pronounced at neutral $\mathrm{pH}$, where the proton concentrations are small. In the case of a sodium phosphate buffer, the $\mathrm{pH}$ decreases by over three units between $0{ }^{\circ} \mathrm{C}$ and $-30{ }^{\circ} \mathrm{C}$. In the case of citrate, the $\mathrm{pH}$ decreases by less than one unit, whereas the $\mathrm{pH}$ of a Tris- $\mathrm{HCl}$ buffer increases by about one unit. ${ }^{60}$ The $\mathrm{pH}$ of the protein samples was set to 4.9 using MES buffer unless indicated otherwise. This low pH has been used in previous NMR structure determinations of this protein. ${ }^{44}$ It minimizes the potential for a change in $\mathrm{pH}$ upon cooling to sub-zero temperature. 
W-band ED-EPR spectra of the S114C and G147C mutants labeled with C7 and C8 are shown in Fig. 2. The two mutants displayed quite different EPR spectra, and while for G147C the C7 and C8 tags had very similar spectra, they were different for S114C for both the central transition (Fig. 2a) and the other transitions broad background (Fig. 2b). In general, the central transition spectra exhibited two shoulders on either side of a narrow feature (marked with a star in Fig. 2a) and the main difference between these spectra is the extent of the separation between the two shoulders. The spectra of the protein samples differ significantly from the spectra of the corresponding free tags in a $\mathrm{D}_{2} \mathrm{O}$ /glycerol mixture (no buffer, in $\mathrm{D}_{2} \mathrm{O}$, bottom trace). The spectra of the free $\mathbf{C 7}$ and $\mathbf{C 8}$ tags are broadly similar, and they are narrower than those of the protein samples and featureless (Fig. 2a). The paramagnetic NMR spectra of proteins labelled with $\mathbf{C 7}$ or $\mathbf{C 8}$ were found to be sensitive to $\mathrm{pH}$, which was attributed to deprotonation of one of the $\mathrm{OH}$ groups of the pendants of the tags. ${ }^{39}$ Therefore, to understand the origin of the different line-shape of the free tags and the labeled proteins we investigated the effect of $\mathrm{pH}$ on the ED-EPR spectra of the free $\mathbf{C 7}$ and $\mathbf{C 8}$ tags. The $\mathbf{C 7}$ and $\mathbf{C 8}$ spectra at pH 3, shown in Fig. 2, are the same but they are different than those of the free tags without buffer and of the protein samples. We found the ED-EPR spectra to change little in the $\mathrm{pH}$ range 3-8 (SI, Fig. S3, S4) adjusted by phosphate/citrate buffers (Table S1). A significant change, manifested in extensive broadening, was detected only at pH 9 (SI, Fig. S3d, 4a), which can be explained by the absence of citrate from the buffer and deprotonation of one of the OH groups. Considering the $\mathrm{pH}$ change with temperature of these buffers, it is reasonable to assume that the $\mathrm{pH}$ value of the $\mathrm{pH}=9 \mathbf{C 7}$ and $\mathbf{C 8}$ samples decreased upon freezing to 5-6, ${ }^{60}$ yet their EPR spectra are considerably broader than those of the C7 and $\mathbf{C 8}$ samples in no buffer having a higher pH (6.7 \pm 0.2$)$. Hence, we conclude that the ED-EPR spectra of the C7 and C8 tags are sensitive to both $\mathrm{pH}$ and buffer conditions, consistent with the sensitivity of (S)-THP and (R)THP contrast agents to inner- and outer-sphere interactions of the metal with coordinating counter anions. ${ }^{42}$

As the tags are enantiomers of each other, the free $\mathbf{C 7}$ and $\mathbf{C 8}$ tags would be expected to show identical ED-EPR spectra. Based on the above results the small differences observed experimentally may have arisen from slightly different salt concentrations in the preparations. 
a)

b)

Figure 2. ED-EPR spectra recorded at W-band and at $10 \mathrm{~K}$ of the $\mathbf{C 7}$ (in black) and $\mathbf{C 8}$ (in red) tags, in the free state and after ligation with the ERp29 mutants. (a) Zoom into the spectral region of the central transition. Spectra were aligned to match the position of the narrow feature marked with a star. The dashed lines mark the positions of the maxima in the spectra of the G147C mutants labeled with the C7 and C8 tags. (b) Display of the full spectral range recorded.

To understand the different ED-EPR line-shapes we carried out spectral simulations. The ED-EPR spectra could not be simulated using the previously reported approach to reproduce the featureless spectra of compounds based on DOTA-Gd(III), ${ }^{24,61}$ where the $D$ distribution is given by two Gaussians of identical width, centered around $D_{c}$

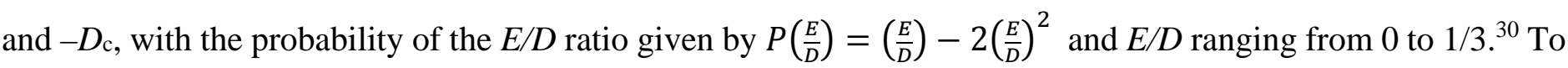
simulate the spectra, we resorted to particular $D$ and $E$ values and included strains. The $D$ and $E$ distributions were taken as two uncorrelated Gaussians around the center values of $D$ and $E$ with a width (full-width at halfmaximum) given by the strain. Figs. 3 and S5 in SI show the simulations of the central transition region and the full spectrum, respectively, using the simulation parameters listed in Table 1. The spectrum of the C7 tag in water/glycerol was also recorded at $240 \mathrm{GHz}$ and is shown in Fig. 3g along with the simulated spectrum using the same parameters as for the W-band spectrum. At this high frequency, the central transition is depleted at low temperatures $(5 \mathrm{~K})$ and the spectrum is dominated by transitions involving the low-lying energy levels, forming the broad background. The simulations indicate that the $D$ value is smallest in the free tags, as suggested by the narrower central peak. In addition, the rhombicity parameter $(\eta=E / D)$ is larger for these spectra, as indicated by the featureless central transition. For the protein samples, $D$ is larger and $\eta$ is smaller. For comparison, the EPR spectrum of DOTA-Gd(III) was simulated with the distribution function described above using $D_{\mathrm{c}}=644 \mathrm{MHz}$, which is a value much smaller than those listed in Table 1 for the $\mathbf{C 7 / C 8}$ tags. $^{58}$ 
a) $\mathbf{C 8}$

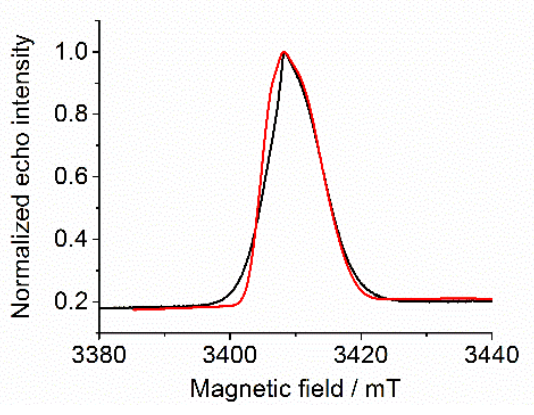

d) $\mathbf{C 7}$

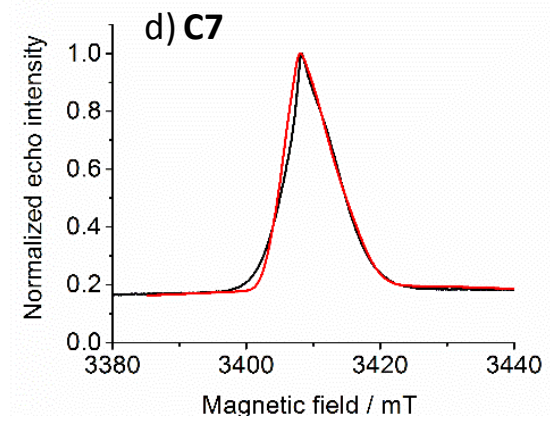

b) S114C-C8

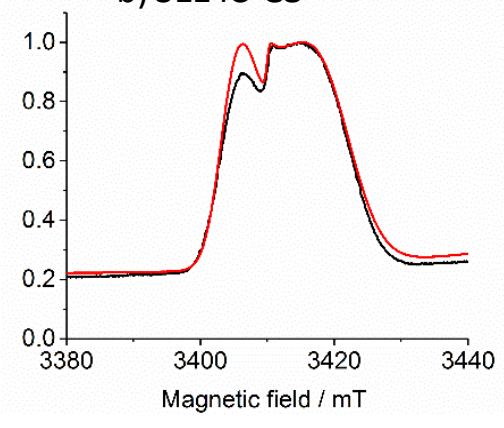

e) S114C- C7

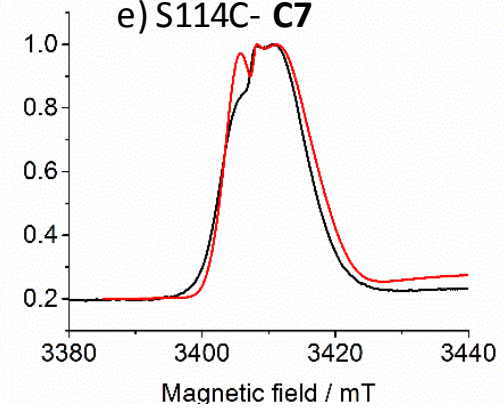

g) $\mathbf{C 7}(240 \mathrm{GHz})$

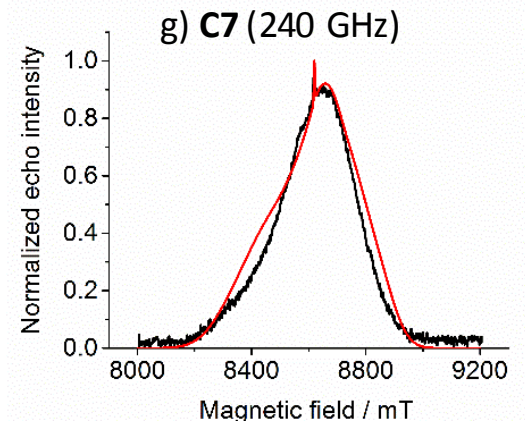

c) G147C- C8
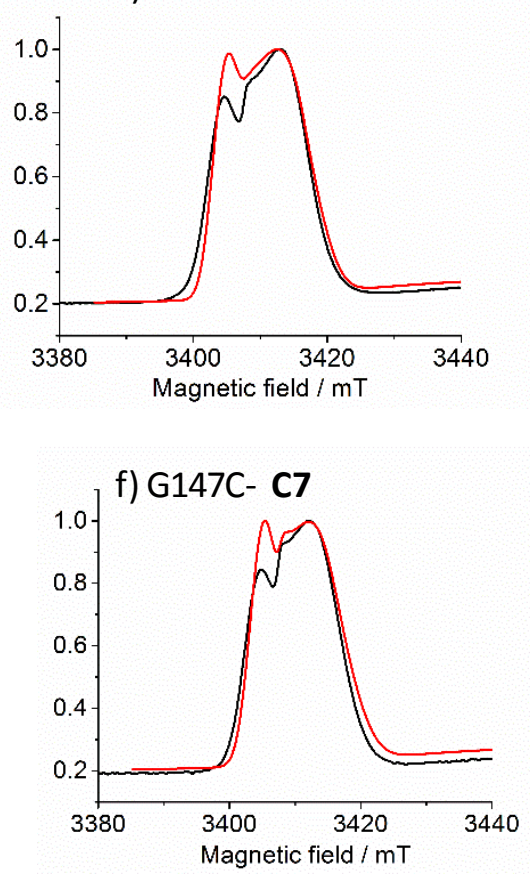

Figure 3. Experimental (black) and simulated (red) EPR spectra of the central transition region of (a) the free C8 tag in $\mathrm{D}_{2} \mathrm{O}$ (no buffer), (b) the mutant S114C with C8 tag, (c) the mutant G147C with C8 tag, (d) the free C7 tag in $\mathrm{D}_{2} \mathrm{O}$, (e) the mutant S114C with C7 tag, (f) the mutant G147C with C7 tag, and (g) the C7 tag in $\mathrm{D}_{2} \mathrm{O}$ (no buffer) at $240 \mathrm{GHz}$ (full spectrum). All spectra were recorded at $10 \mathrm{~K}$ except (g) which was measured at $5 \mathrm{~K}$. The simulation parameters are given in Table 1.

Simulations of the spectra of the free tags at different $\mathrm{pH}$ values (SI, Fig. S3) revealed an increase in $D$ with an unchanged or little reduced value of $\eta$ compared to the measurements in buffer-free water/glycerol (Table 1). Regardless of the $\mathrm{pH}$ (except for the $\mathrm{pH}=9$ ), the $D$ value was smaller and $\eta$ was larger in the free tags than in the protein samples. When the $\mathrm{pH}$ of the ERp29 S114C-C8 sample was adjusted to $\mathrm{pH} 8$ by switching from MES 
buffer to Tris-HCl buffer, the width of its spectrum decreased (SI, Fig. S6). This effect was unexpected, as the free C8 tag showed a much broader central transition region at high $\mathrm{pH}$ (Fig. S3d). This can arise from the presence of different and/or to associated changes in the tag's local environment.

In summary, the EPR spectra of the free $\mathbf{C 7}$ and $\mathbf{C 8}$ tags are very similar, but sensitive to increased $\mathrm{pH}$ and the presence of ions in the solution, which tend to increase $D$ and reduce $\eta$. Following conjugation to ERp29, the EPR spectra further changed, exhibiting different spectra for the two mutants. Only one of the mutants (S114C) showed significant differences between the C7 and C8 tags. These results suggest that the ZFS is affected by local charges in the protein. To investigate the local structural features responsible for altering ZFS parameters, we carried out ENDOR measurements as described below.

Table 1. Parameters Used to Simulate the EPR Spectra of the Samples Studied

\begin{tabular}{|l|l|l|l|l|}
\hline & $\boldsymbol{D}(\mathbf{M H z})$ & $\boldsymbol{\eta}=\boldsymbol{E} / \mathbf{D}$ & $\begin{array}{l}\text { Dstrain } \\
(\mathbf{M H z})\end{array}$ & $\begin{array}{l}\text { Estrain } \\
(\mathbf{M H z})\end{array}$ \\
\hline C8 & -1500 & 0.2 & 450 & 350 \\
\hline C7 & -1500 & 0.23 & 850 & 250 \\
\hline C8, pH = 3 & -1600 & 0.200 & 450 & 350 \\
\hline C7, pH = 3 & -1650 & 0.182 & 450 & 300 \\
\hline G147C-C8 & -1800 & 0.15 & 250 & 350 \\
\hline G147C-C7 & -1800 & 0.15 & 450 & 350 \\
\hline S114C-C7 & -1800 & 0.15 & 650 & 150 \\
\hline S114C-C8 & -2074 & 0 & 820 & 550 \\
& -2100 & 0.15 & 550 & 250 \\
\hline
\end{tabular}

${ }^{a}$ These two sets of values provide similar fit quality. The simulations shown in Fig. 3 were obtained with the top values.

\section{$\underline{\text { ENDOR measurements }}$}

Recent ${ }^{1} \mathrm{H}$-ENDOR measurements carried out on DOTA-Gd(III) revealed that the hyperfine coupling with ${ }^{1} \mathrm{H}$ nuclei can be described by the point-dipole approximation using the Gd-H distances from the crystal structure. ${ }^{58}$ Accordingly, we expected that ${ }^{1} \mathrm{H}$-ENDOR measurements of the free and protein-bound tags would reveal structural differences that may explain the observed differences in the ZFS. For the free tags in just $\mathrm{D}_{2} \mathrm{O}$, the ENDOR measurements were carried out at the magnetic field corresponding to the maximum of the EPR spectrum (Fig. 2a, bottom traces). For the protein samples, where the EPR spectrum exhibits two local maxima, the ENDOR 
measurements were performed at the magnetic field corresponding to these two maxima, referred to as the highfield and low-field positions. At these magnetic fields, the major contribution to the echo comes from the central $|-1 / 2\rangle \rightarrow|1 / 2\rangle$ transition, the contributions from the $|-3 / 2\rangle \rightarrow|-1 / 2\rangle$ and $|1 / 2\rangle \rightarrow|3 / 2\rangle$ transitions are much smaller, and those of the other transitions can practically be neglected (SI, Fig. S7). All samples were prepared in $\mathrm{D}_{2} \mathrm{O}$ so that only non-exchangeable protons contribute to the ${ }^{1} \mathrm{H}$-ENDOR spectra.

The ENDOR spectra of all samples are presented in Fig. 4. The spectra of the free $\mathbf{C 7}$ and $\mathbf{C 8}$ tags are very similar, but there are interesting differences compared to the corresponding spectrum of DOTA-Gd(III) (Fig. 4a). For DOTA-Gd(III), the three main doublet features arise from two groups of 12 protons situated 3.7 and $4.4 \AA$ from the Gd(III) ion, with the perpendicular component of the dipolar interaction, $T_{\perp}$, given by $1.55 \mathrm{MHz}$ (red arrow in Fig. 4a) and $0.91 \mathrm{MHz}$ (blue arrow, Fig. 4a), respectively. ${ }^{58}$ The main contribution comes from the $\pm 1 / 2$ manifolds, while the ENDOR signals originating from the $\pm 3 / 2$ manifolds are small and give rise to signals overlapping with the peaks around $\pm 1.5 \mathrm{MHz}$, as manifested by the somewhat more intense signal at $1.5 \mathrm{MHz} .^{58}$ The spectra of the $\mathbf{C 7}$ and $\mathbf{C 8}$ tags also show very minor asymmetry with respect to $v_{\mathrm{RF}}-v_{\mathrm{H}}=0$, indicating that they arise predominantly from the $\pm 1 / 2$ manifolds. The asymmetry, manifested mainly in the larger intensity at $+1.8 \mathrm{MHz}$, is due to contributions from other spin manifolds. The number of spectral features increases from 3 to 6 as there are more inequivalent protons in $\mathbf{C 7}$ and $\mathbf{C 8}$ compared to DOTA-Gd(III). The low-field ENDOR spectra of the protein samples are practically identical (Fig. 4b, SI Fig. S8a), whereas the high-field spectra show some minor differences between S114C-C7 and S114C-C8 in the relative intensities of the peaks in the $\pm(0.5-0.9) \mathrm{MHz}$ region (Fig. 4c, SI, Fig. S6b).

There are small differences between the spectra recorded at the low-field and high-field positions, with the spectra recorded at the high-field position exhibiting a higher resemblance to the spectra of the free tags (Fig. 4a). We attribute the differences between the low-field and high-field spectra to orientation selection, which is somewhat more pronounced at the low-field position (see SI, Fig. S9 for details). Orientation selection becomes more significant as $D$ increases and $E / D$ decreases.. In addition, the spectra are less symmetric with respect to $v_{\mathrm{RF}}-v_{\mathrm{H}}$ $=0$, as compared with the spectra of the free tags and DOTA-Gd(III). The increased asymmetry arises from larger relative contributions from transitions other than the central transition, which increases with the width of the central line (see SI, Fig. S7). ${ }^{62}$ 
a)

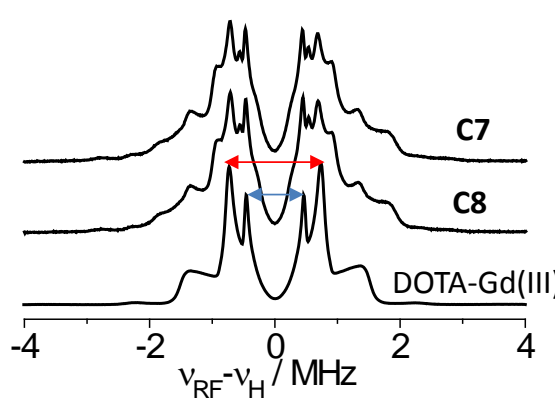

b)

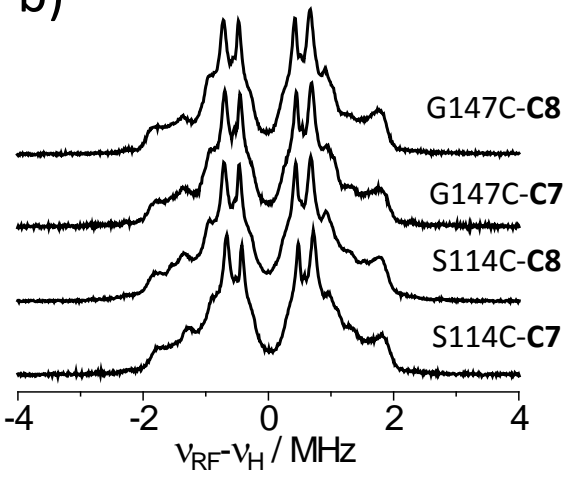

c)

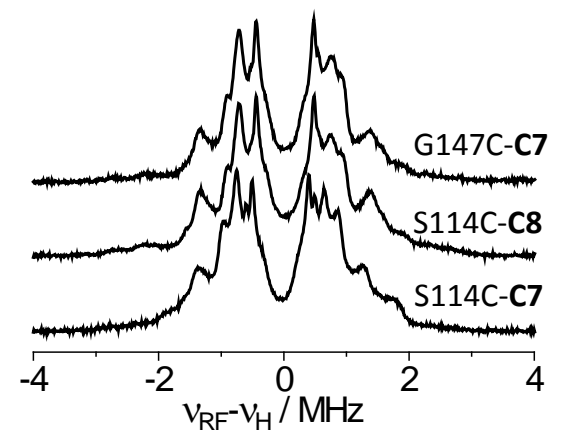

Figure 4. Experimental ${ }^{1} \mathrm{H}$-ENDOR spectra of the free and ligated $\mathbf{C 7}$ and $\mathbf{C 8}$ tags. (a) ENDOR spectra of free C7 and C8 tags (without buffer) compared with the corresponding spectrum of DOTA-Gd(III). The arrows mark the $T_{\perp}=1.55 \mathrm{MHz}$ (red) and $T_{\perp}=0.91 \mathrm{MHz}$ doublets (blue). (b) ENDOR spectra of G147C-C8, G147C-C7, S114C-C8, and S114C-C7 measured at the low-field position. (c) Same as (b) but measured at the high-field position. The spectrum of G147C-C8 was not recorded in view of the similarity of the spectra between $\mathbf{C 7}$ and C8 tags observed in (b).

Mims ${ }^{2} \mathrm{H}-\mathrm{ENDOR}$ spectra of the exchangeable protons of G147C-C8 at high and low fields again revealed clear evidence for orientation selection, with an asymmetry suggesting contributions from spin manifolds other than the $\pm 1 / 2$ manifolds (Fig. 5). The spectra show three types of signals, one corresponding to distant water, with $A$ $=0.11 \mathrm{MHz}$, and features corresponding to $A=0.36-0.50$ and $A=0.76-0.88 \mathrm{MHz}$. Additional fine structure could arise from quadrupole splitting. $T_{\perp}=2.58 \mathrm{MHz}$ was reported for protons in the Gd(III) aquo-complex, ${ }^{63}$ corresponding to $T_{\perp}=0.4 \mathrm{MHz}$ for ${ }^{2} \mathrm{H}$. Therefore, we assign the lines with $A=0.36-0.50$ to $T_{\perp}$ of a water ligand and/or the coordinated $\mathrm{OH}$ groups, whereas the features at $0.76-0.88 \mathrm{MHz}$ are assigned to the corresponding $\mathrm{T}_{\|}$ features overlapping with contributions of deuterons with smaller $T_{\perp}$ values from $\pm 3 / 2$ manifolds.

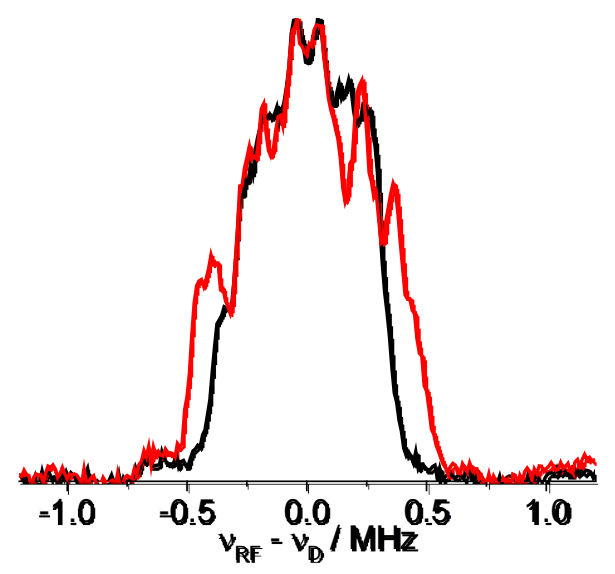

Figure 5. W-band Mims ${ }^{2} \mathrm{H}-\mathrm{ENDOR}$ spectra of G147C-C8 measured at the high-field position (red) and lowfield position (black). 
To better understand the ${ }^{1} \mathrm{H}$-ENDOR spectra we carried out spectral simulations. We followed the approach used in the simulations of the Davies ${ }^{1} \mathrm{H}$-ENDOR spectrum of DOTA-Gd(III). ${ }^{58}$ While there is no crystal structure for C7 or C8, the crystal structure of Eu(III) with $(S)$-THP, the parent molecule of $\mathbf{C 7}$ and $\mathbf{C 8}$, has been reported. ${ }^{64}$ Accordingly, we used the Eu-H distances from the (S)-THP-Eu(III) crystal as initial values in the simulations of the ${ }^{1} \mathrm{H}$-ENDOR spectra (see Fig. S10). We then grouped the different non-exchangeable hydrogens into four classes according to their distances from the Gd(III) (Fig. 6a and Table 2). Class B and C protons have couplings similar to those in the two groups in DOTA-Gd(III). ${ }^{58}$ The simulated ENDOR spectrum was obtained by summing the individual ENDOR spectra of the different classes weighted by the number of hydrogens in each class. Because all ENDOR spectra were practically the same in terms of peaks position, and any differences were small and mostly affecting relative intensities, the same four classes were used for all samples. We started by simulating the ENDOR spectra of the free tags shown in Fig. 4a, where orientation selection is not evident, considering only contributions from the central transition, namely only ENDOR arising from the $m_{\mathrm{S}}= \pm 1 / 2$ manifolds. The simulated spectra for the $\mathbf{C} \mathbf{8}$ tag gave a reasonable fit to the experimental spectrum, except for a reduced intensity at $\pm 0.7 \mathrm{MHz}$ and some missing intensity in the $\pm 2 \mathrm{MHz}$ peaks along with the associated asymmetry (Fig. 6b). The simulations of the high- and low-field ENDOR spectra of the protein samples are given and discussed in the SI (Figs. S11, S12, S13).

Table 2. The Classes of Protons used to Simulate the Davies ${ }^{1} \mathrm{H}$-ENDOR Spectra.

\begin{tabular}{|c|c|c|c|c|}
\hline Proton class $^{\mathbf{a}}$ & $\boldsymbol{T}_{\perp}(\mathbf{M H z})^{\mathbf{b}}$ & Distance $(\AA)$ & Weight & ${\text { Euler angle } \boldsymbol{\beta}^{\mathbf{c}}}^{\text {( })}$ \\
\hline A (red) & 1.91 & 3.46 & 4 & $60^{\circ}$ \\
\hline B (green) & 1.45 & 3.79 & $9^{\mathrm{d}}$ & $40^{\circ}$ or $90^{\circ}$ \\
\hline C (blue) & 0.95 & 4.37 & $15^{\mathrm{d}}$ & $40^{\circ}$ \\
\hline D (magenta) & 0.6 & 5.09 & 11 & $50^{\circ}$ \\
\hline
\end{tabular}

${ }^{\mathrm{a}}$ The color codes correspond to the colors used in Fig. 6a.

${ }^{\mathrm{b}} T_{\perp}=\frac{g_{e} g_{n} \beta_{e} \beta_{n}}{h r^{3}}$, where $g_{\mathrm{e}}$ and $g_{\mathrm{n}}$ are the electron and nuclear spin $g$ values, $\beta_{\mathrm{e}}$ and $\beta_{\mathrm{n}}$ are the corresponding Bohr magnetons, $h$ is the Planck constant, and $r$ is the electron nuclear distance.

c The Euler angles refer to the Gd-H vector with respect to the principal axis of the ZFS. It is needed for calculating orientation selection ENDOR spectra, see SI.

${ }^{\mathrm{d}}$ According to the molecular structure, class B should have 8 protons and class C 16 protons; 9 and 15 gave somewhat better agreement with the experimental spectra. 
a)
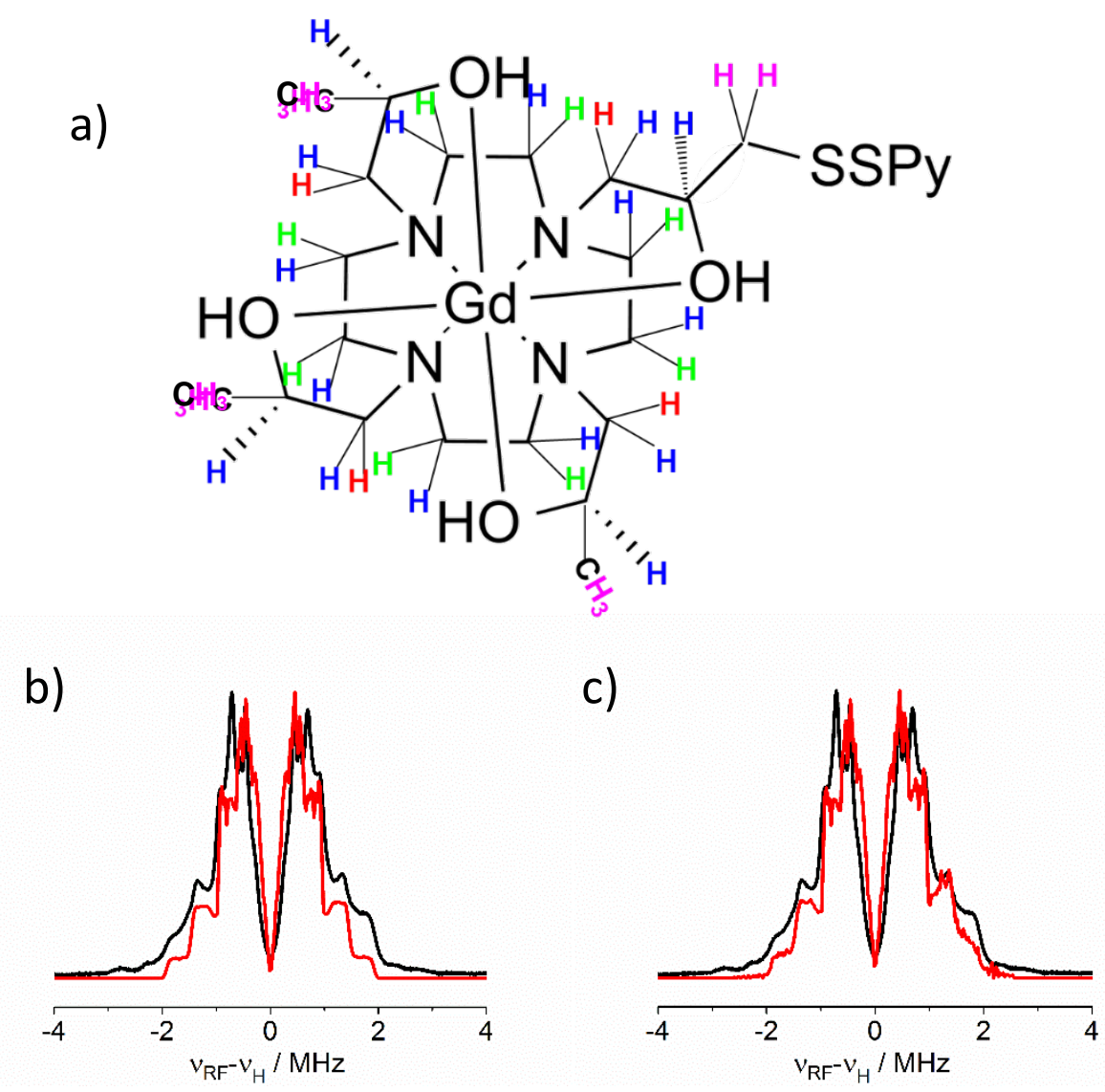

Figure 6. Simulations of Davies ${ }^{1} \mathrm{H}-\mathrm{ENDOR}$ spectra of the $\mathbf{C 8}$ tag without buffer. (a) Four classes of hydrogens in the C8 tag color-coded as follows: A - red, B - green, C - blue, D - magenta (Table 2). (b) Experimental (black) and simulated (red) Davies ${ }^{1} \mathrm{H}$-ENDOR spectra of the $\mathbf{C 8}$ tag considering only the $\pm 1 / 2$ manifolds and (c) with the addition of contributions from the $\pm 3 / 2$ manifold for the $\mathrm{C}$ - and D-class protons (relative weights are $50 \%$ for the $-3 / 2$ manifold and $12.5 \%$ for the $+3 / 2$ manifold). In all these simulations $\beta=40^{\circ}$ was used for class B.

As mentioned earlier, the asymmetry in the intensities of the doublets at $\pm 2 \mathrm{MHz}$ suggests contributions from manifolds other than the $\pm 1 / 2$ manifolds, mostly from the $-3 / 2$ manifold, but also from the $+3 / 2$ manifold. These contributions are relatively larger for the samples with a broader central transition (Fig. S7). They are particularly crucial for the protons with the small couplings, namely classes C and D. The inclusion of these manifolds produces peaks around $\pm 2 \mathrm{MHz}$ (Fig. S14). The other protons with the larger couplings contribute broader, mostly unresolved powder patterns that are evident at the wings of the spectra. A better fit could be obtained for the free tags by including small contributions from these transitions for the class C- and D- protons, as shown in Fig. 6c for C8. The simulations of all other samples are given in the SI (Figs. S15, S16). While we could reproduce the majority of the features of the ENDOR spectra, mostly in terms of peak positions, we could not closely reproduce the asymmetry observed in the spectra and the small but consistent differences between the low- and high-field spectra at the edges of the spectra. This could be due to several simplifications made to reduce the number of parameters involved, such as not taking into account the full $D$ and $E$ distributions in the determination of the 
selected orientations, assuming a single $\beta$ value for all protons in a group rather than a distribution of $\beta$ values, as well as setting one relevant Euler angle to zero and ignoring the contributions of manifolds other than $\pm 1 / 2$ manifolds for proton classes A and B.

In summary, the ENDOR spectra of the non-exchangeable protons in all samples can reasonably be accounted for by a superposition of the four groups of protons present in the structure of the tags. The spectra of the protein samples revealed orientation selection effects, which could be partially accounted for in the simulations. The similarity of the ENDOR data of the C7 and C8 tags indicates that the conformations of the respective cyclen rings are mirror images of each other, following the chiralities of the pendant arms. Furthermore, they were much less sensitive to the subtle structural changes occurring between free and bound tags than the ZFS manifested in the ED-EPR spectra.

\section{$\underline{\text { DEER measurements }}$}

DEER measurements were carried out on the S114C and G147C mutants labeled with the C7 and C8 tags (Fig. 7). Of particular interest are the results of S114C-C8 and S114C-C7, which exhibit no significant differences between the distance distributions, while the ED-EPR spectra were notably different. This indicates independence of tag chirality (Fig. 7). The DEER distance distributions of G147C-C8 and G147C-C7 are broader and differ in the intensity around 4-5 nm appearing for G147C-C7. Considering the lower SNR of the latter DEER trace, we attribute the shoulder at 4-5 nm to SNR limitations. Similar to previous results obtained with the $\mathbf{C} \mathbf{1}^{16}$ and $\mathbf{C} \mathbf{9}^{12}$ tags for the same ERp29 mutants, the S114C mutant gave a significantly narrower distance distribution than the G147C mutant, reflecting the location of residue 147 at a site of the protein that allows greater conformational freedom of the tag. ${ }^{12}$ Using the same observe and pump frequencies with the field shifted by 8 mT gave the same results, indicating the absence of any resolved orientation dependence. Similarly, the same distance distributions were obtained from measurements carried out with a dual-mode cavity and setting the difference between the pump and observe frequencies to $\Delta v=230 \mathrm{MHz}$ (Fig. S18a). The distance distributions were also insensitive to changes in $\mathrm{pH}$; a S114C-C8 sample prepared at $\mathrm{pH}=8$ (uncorrected $\mathrm{pH}$ meter reading) gave a similar distance distribution (Fig. S18b).

It is interesting to compare the DEER data of S114C-C7 and S114C-C8 with those of the same protein mutants labeled with the $\mathbf{C 9}$ tag. ${ }^{12}$ All three tags feature the same chemical linkage between the cyclen ring and the protein, which results in a short tether and limited tag flexibility and similarly narrow widths of the distance distributions (about 0.4-0.5 nm). For comparison, the corresponding width of the distance distribution achieved for S114C labeled with MTSL (chemical structure is given in Fig. S1) was $0.7 \mathrm{~nm}^{23}$ The maximum of the distance distribution measured in the present work is $6.0 \mathrm{~nm}$ compared to $5.8 \mathrm{~nm}$ measured with the $\mathbf{C 9}$ tag. The small size of this difference suggests that the bulky phenylethylacetamide pendant arms in the C9 tag, which were 
designed to restrict cyclen isomerization as well as introduce steric hindrance, affect neither the width nor the maximum of the distance distribution. This is in stark contrast to the ZFS, which is significantly affected by the replacement of three amide oxygens with three $\mathrm{OH}$ oxygens (Fig. 1).
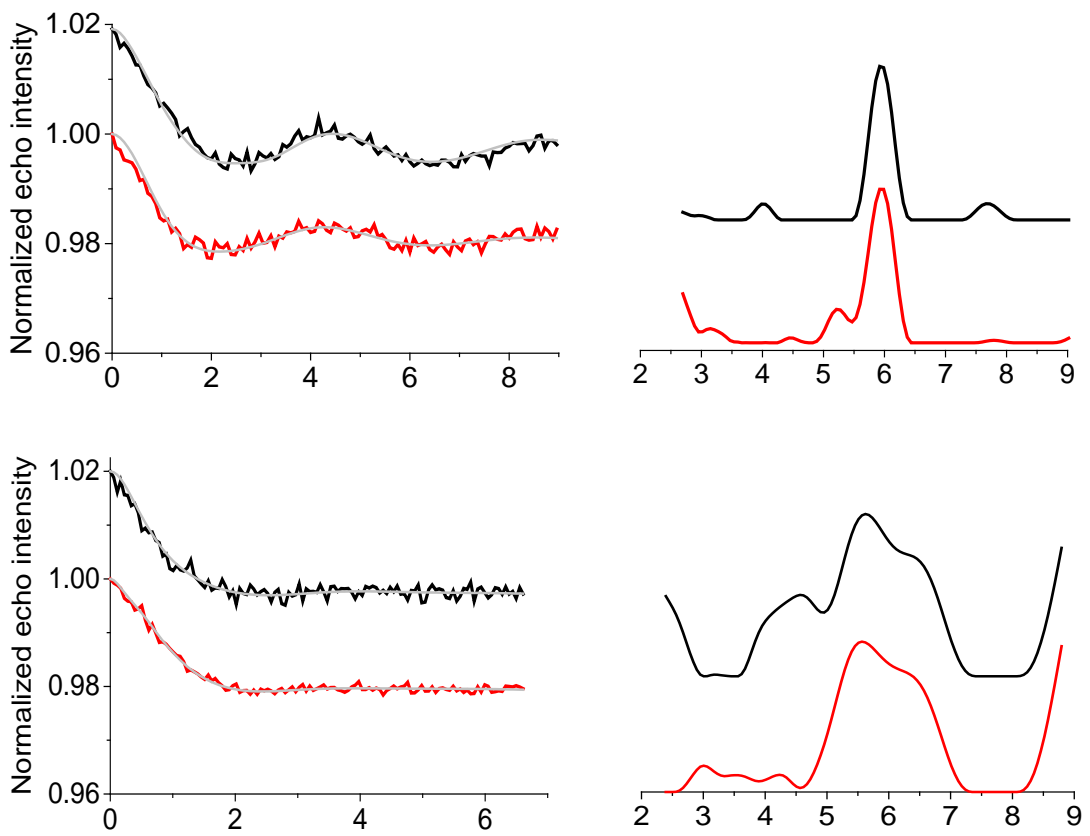

Figure 7. DEER distance measurements in ERp29 mutants ligated with the $\mathbf{C 7}$ or $\mathbf{C 8}$ tags. (a) DEER traces after background removal of S114C ligated with the $\mathbf{C 7}$ or $\mathbf{C 8}$ tags. The grey line corresponds to the fitted trace obtained with the distance distribution shown in (b). (b) Corresponding distance distributions. Regularization parameters were 5 for C7 and 20 for C8. (c) and (d) Same as (a) and (b), respectively, except for the G147C mutant. Regularization parameters were 35 for $\mathbf{C 7}$ and 50 for C8. The primary DEER data are shown in Fig. S17.

\section{Discussion}

The present work shows that the two enantiomers of a new cyclen-based Gd(III) tag, $\mathbf{C 7}$ and $\mathbf{C 8}$, which feature a short chemical linker to the protein and provide eight coordination sites for the Gd(III) ion via four nitrogen atoms and four $\mathrm{OH}$ groups, present more involved EPR properties than previously designed cyclen tags, while being eminently suited for DEER distance measurements. Attached to two single-cysteine mutants, S114C and G147C, of the homodimeric protein ERp29, the EPR properties of the $\mathbf{C 7}$ and $\mathbf{C 8}$ tags can be compared to those of the $\mathbf{C} 1^{16}$ and $\mathbf{C 9}{ }^{12}$ tags (Fig. 1) investigated previously with the same mutants of ERp29. Importantly, the distance distributions obtained from DEER experiments with the $\mathbf{C 7}$ and $\mathbf{C 8}$ tags were similarly narrow as those obtained with the $\mathbf{C 9}$ tag, which is one of the best tags for obtaining narrow Gd(III)-Gd(III) distance distributions with DEER for distances below $4 \mathrm{~nm} \cdot{ }^{12}$ No significant difference was detected between the $S(\mathbf{C 7})$ and $R(\mathbf{C 8})$ stereoisomers. The modulation depth (about 1.5-2\%) was less than that obtained with the $\mathbf{C 9}$ and $\mathbf{C 1}$ tags, which 
was about 5-6\%, but not as shallow as expected in view of the EPR line-width and the labeling efficiency. Comparison of the central transition region of the ED-EPR spectra of different cyclen tags (C1, C8, C9 and T2; Fig. 1) attached to different proteins (ERp29 S114C and G147C mutants, T4 lysozyme) highlights the large difference in line-widths of the central transitions and shows that, in the case of the $\mathbf{C} \mathbf{8}$ tag, the central transition region greatly exceeds the bandwidth of a 15 ns pump pulse in the DEER experiment (Fig. 8). It may be that the broad line-width relaxes some of the incompletely understood effects of the dipolar pseudo-secular terms on the modulation depth. ${ }^{25}$

An unexpected result of this work is the dependence of the EPR spectra of the $\mathbf{C 7}$ and $\mathbf{C 8}$ tags on the labeling site of the protein. While the W-band ED-EPR spectra of the free tags in $\mathrm{D}_{2} \mathrm{O} /$ glycerol $(\mathrm{pH}=6.7 \pm 0.2$ uncorrected) are similar to each other and display a mostly featureless central-transition line-shape, the line-shape varies as a function of buffer. The S114C-C8 sample also displayed a different line-shape between samples prepared in Tris$\mathrm{HCl}$ buffer at $\mathrm{pH} 8.0$ and MES buffer at $\mathrm{pH} 4.9$ (Fig. S6). The differences may be a consequence of direct interactions with buffer anions, the result of different $\mathrm{pH}$ values, and changes in the local protein environment, all affecting the charge states of the $\mathrm{OH}$ groups of the pendant arms of the tag. Involvement of the $\mathrm{OH}$ protons of the pendant arms in H-bonding with buffer anions has been reported earlier for (S)-THP-Eu(III) complexes $^{42}$ to affect the effective charge on the oxygen ligands. An asymmetric charge distribution about the Gd(III) ion is expected to change the ZFS tensor, and consequently the $D$ parameter. Following ligation with the cysteine mutants of ERp29, significant differences in the EPR line-shape of C7 and C8 were found only for S114C. It is possible that this effect is caused by the side chain of Glu 113, which is near the side chain of residue $114{ }^{44}$ Residue 147 does not have a charged side chain nearby. Like in previous results obtained with the $\mathbf{C 9}$ tag, ${ }^{12}$ the S114C mutant showed a considerably narrower distance distribution than the G147C mutant, reflecting the location of the site in a structurally less exposed region of the protein, which limits the conformational space accessible to the tag.

It is interesting to compare the spectra and ZFS associated with the $\mathbf{C 7}$ and $\mathbf{C 8}$ tags, which contain four OH pendant arms, to those of (i) the $\mathbf{C 1}$ tag, which has amide pendant arms and no OH group, (ii) the $\mathbf{C 9}$ tag, which has one $\mathrm{OH}$ and three amide groups, and (iii) the double-arm tags $\mathbf{T 1}$ and T2, which have two OH and two amide pendant arms (Figs 1 and 8). Most notably, the line-width and, therefore, the ZFS increase with the number of $\mathrm{OH}$ groups (Table 3) and so does the sensitivity of the EPR spectrum to the labeling site. In the case of the T1 and T2 tags, some minor differences in the W-band ED-EPR spectrum were reported for different protein ligation sites, ${ }^{11}$ but the interpretation of these results was complicated by the fact that two tags were ligated to different sites of the protein, as opposed to dimeric ERp29, where only a single labeling site is needed for DEER measurements. While this work shows that the $\mathbf{C 7}$ and $\mathbf{C 8}$ tags have the potential of reporting on local structural features such as charge distribution, much more work is needed for this to become a reliable source of information 
and to disentangle the effect of anion binding from the protonation state of the hydroxyl pendants. In particular, the variability of the EPR spectrum must be explored for more proteins and sites, preferably including a systematic variation of the hydrophobicity of the environment as well as the presence of different ions in the solution. In addition, quantum chemical calculations could contribute significantly to understanding the factors that affect $D$ and $E$, and their distributions. We are aware that such calculations currently pose a great challenge but there are recent attempts to deal with this. ${ }^{65}$ With regard to DEER applications, we prefer the $\mathbf{C} \mathbf{9}$ tag for distances longer than $4 \mathrm{~nm}$, where sensitivity is important and dipolar pseudo-secular terms can be neglected, but the C7 and C8 tags will be much better for short distances as the pseudo-secular terms can be neglected for tags with a large ZFS. The compromise in sensitivity is not detrimental for measurements of short distances, as they can be conducted with short dipolar evolution times.

Figure 8. ED-EPR spectra of the samples indicated in the figure. The red trace indicates the bandwidth of a pump pulse of 15 ns.

Finally, while the powder pattern of the ${ }^{1} \mathrm{H}$-ENDOR spectra of the non-exchangeable protons showed some evidence for orientation selection, the spectra were not very sensitive to subtle changes in the local environment of the Gd(III) ion, suggesting the absence of significant geometrical changes in the cyclen ring and more indirect effects from the large variations in the ZFS associated with the $\mathbf{C 7}$ and $\mathbf{C 8}$ tags in different environments. 
Table 3. Comparison of ZFS Parameters Obtained from the Simulations of the W-band ED-EPR Spectra of Figure 8 as Shown in Fig. S19.

\begin{tabular}{|l|l|l|l|l|l|}
\hline Sample & $\begin{array}{l}\text { Width of central } \\
\text { transition } \mathbf{~}^{\mathbf{m} T}\end{array}$ & $\mathbf{D , ~ M H z}$ & E/D & Dstrain, MHz & Estrain, MHz \\
\hline ERp29-S114C-C1 & 1.15 & -470 & 0 & 840 & 375 \\
\hline ERp29-S114C-C9 & 2.48 & -575 & 0.45 & 230 & 550 \\
\hline T4L-B-T2 & 9.26 & -1850 & 0 & 1330 & 0 \\
\hline ERp29-S114C-C8 & 15.50 & -2074 & 0 & 820 & 550 \\
& & -2100 & 0.15 & 550 & 250 \\
\hline
\end{tabular}

${ }^{a}$ Full-width at half-height of the central transition only.

${ }^{b}$ These two sets of values provide similar fit quality. The simulations shown in Fig. 3 were obtained with the top values.

\section{Conclusions}

This work evaluated the performance of two new compact and hydrophilic Gd(III) tags, C7 and C8, as spin labels for W-band DEER distance measurements. The two tags are enantiomers of the same chemical structure. Tested on the mutant S114C of ERp29, which is known to give a narrow distance distribution with the C9 tag, both the $\mathbf{C 7}$ and $\mathbf{C 8}$ tags reported a similar narrow distance distribution as the $\mathbf{C 9}$ tag. Due to their much broader central EPR transition, the $\mathbf{C 7}$ and $\mathbf{C 8}$ tags will be superior for measurements of short $(<4 \mathrm{~nm})$ distances. A particularly interesting and unique property of these tags is the sensitivity of their EPR spectra to their nearby protein environment, as evidenced by very different ED-EPR spectra for tags attached to the ERp29 mutants S114C and G147C. Even spectra of the free tags proved sensitive to the buffer conditions, whereas ${ }^{1} \mathrm{H}$-ENDOR spectra of the non-exchangeable protons of the tags were largely conserved. It may be possible to develop the sensitivity of the ZFS of the Gd(III) ion in these tags into a tool for probing the local environment of different protein sites, in analogy to established nitroxide labels.

\section{Acknowledgments}

We thank Professors Mark Sherwin and Songi Han for help with measurements at $240 \mathrm{GHz}$. D. G. acknowledges the support of the Israel Science Foundation (ISF grant No. 334/14). J. C. was supported by NSF grant MCB 1617025. This research was made possible in part by the historic generosity of the Harold Perlman Family (D. G.). D. G. holds the Erich Klieger Professorial Chair in Chemical Physics. T. B. acknowledges financial support from the Minerva Foundation. G. O. thanks the Australian Research Council (ARC) for a Laureate Fellowship, B. G. thanks the ARC for a Future Fellowship, and G. O. and B. G. thank the ARC for financial support. 
Supplementary information: Buffer composition, Mass spectra, ED-EPR spectral simulations, additional ED-

EPR spectra, comparison of ENDOR spectra and ENDOR simulations, raw DEER data, and distance distribution

analysis validation.

We declare no conflict of interest.

\section{References}

1. Jeschke, G., DEER distance measurements on proteins. Ann. Rev. Phys. Chem. 2012, 63, 419-446.

2. Jeschke, G.; Polyhach, Y., Distance measurements on spin-labelled biomacromolecules by pulsed electron paramagnetic resonance. Phys. Chem. Chem. Phys. 2007, 9, 1895-1910.

3. Schmidt, T.; Wälti, M. A.; Baber, J. L.; Hustedt, E. J.; Clore, G. M., Long Distance Measurements up to $160 \AA$ in the GroEL Tetradecamer Using Q-Band DEER EPR Spectroscopy. Angew. Chem. Int. Ed. 2016, 55, 15905-15909.

4. Cunningham, T. F.; Putterman, M. R.; Desai, A.; Horne, W. S.; Saxena, S., The double-histidine $\mathrm{Cu}^{2+}$-binding motif: A highly rigid, site-specific spin probe for electron spin resonance distance measurements. Angew. Chem. Intl. Ed. 2015, 127, 6428-6432.

5. Yang, Z. Y.; Kise, D.; Saxena, S., An approach towards the measurement of nanometer range distances based on $\mathrm{Cu}^{2+}$ ions and ESR. J. Phys. Chem. B 2010, 114, 6165-6174.

6. Banerjee, D.; Yagi, H.; Huber, T.; Otting, G.; Goldfarb, D., Nanometer-range distance measurement in a protein using $\mathrm{Mn}^{2+}$ tags. J. Phys. Chem. Lett. 2012, 3, 157-160.

7. Martorana, A.; Yang, Y.; Zhao, Y.; Li, Q.-F.; Su, X.-C.; Goldfarb, D., Mn (II) tags for DEER distance measurements in proteins via C-S attachment. Dalton Trans. 2015, 44, 20812-20816.

8. Ching, H. V.; Demay-Drouhard, P.; Bertrand, H. C.; Policar, C.; Tabares, L. C.; Un, S., Nanometric distance measurements between Mn (II) DOTA centers. Phys. Chem. Chem. Phys. 2015, 17, 23368-23377.

9. Feintuch, A.; Otting, G.; Goldfarb, D., Gd ${ }^{3+}$ spin labeling for measuring distances in biomacromolecules: why and how? Methods Enzymol. 2015, 563, 415-457.

10. Goldfarb, D., Gd $\mathrm{Gd}^{3+}$ spin labeling for distance measurements by pulse EPR spectroscopy. Phys. Chem. Chem. Phys. 2014, 16, 9685-9699.

11. Welegedara, A. P.; Yang, Y.; Lee, M. D.; Swarbrick, J. D.; Huber, T.; Graham, B.; Goldfarb, D.; Otting, G., Doublearm lanthanide tags deliver narrow $\mathrm{Gd}^{3+}-\mathrm{Gd}^{3+}$ distance distributions in double electron-electron resonance (DEER) measurements. Chem. Eur. J. 2017, 23, 11694-11702.

12. Abdelkader, E. H.; Lee, M. D.; Feintuch, A.; Cohen, M. R.; Swarbrick, J. D.; Otting, G.; Graham, B.; Goldfarb, D., A new $\mathrm{Gd}^{3+}$ spin label for $\mathrm{Gd}^{3+}-\mathrm{Gd}^{3+}$ distance measurements in proteins produces narrow distance distributions. J. Phys. Chem. Lett. 2015, 6, 5016-5021.

13. Matalon, E.; Huber, T.; Hagelueken, G.; Graham, B.; Feintuch, A.; Frydman, V.; Otting, G.; Goldfarb, D., Gd ${ }^{3+}$ spin labels for high sensitivity distance measurements in trans-membrane helices. Angew. Chem. Int. Ed. 2013, 5211831 11834

14. Potapov, A.; Yagi, H.; Huber, T.; Jergic, S.; Dixon, N. E.; Otting, G.; Goldfarb, D., Nanometer-scale distance measurements in proteins Using $\mathrm{Gd}^{3+}$ spin labeling. J. Am. Chem. Soc. 2010, 132, 9040-9048.

15. Edwards, D. T.; Huber, T.; Hussain, S.; Stone, K. M.; Kinnebrew, M.; Kaminker, I.; Matalon, E.; Sherwin, M. S.; Goldfarb, D.; Han, S., Determining the oligomeric structure of proteorhodopsin by $\mathrm{Gd}^{3+}$-based pulsed dipolar spectroscopy of multiple distances. Structure 2014, 22, 1677-1686.

16. Yagi, H.; Banerjee, D.; Graham, B.; Huber, T.; Goldfarb, D.; Otting, G., Gadolinium tagging for high-precision measurements of $6 \mathrm{~nm}$ distances in protein assemblies by EPR. J. Am. Chem. Soc. 2011, 133, 10418-10421

17. Abdelkader, E. H.; Feintuch, A.; Yao, X.; Adams, L. A.; Aurelio, L.; Graham, B.; Goldfarb, D.; Otting, G., Protein conformation by EPR spectroscopy using gadolinium tags clicked to genetically encoded p-azido-L-phenylalanine. Chem. Comm. 2015, 51, 15898-15901.

18. Gordon - Grossman, M.; Kaminker, I.; Gofman, Y.; Shai, Y.; Goldfarb, D., W-band pulse EPR distance measurements in peptides using $\mathrm{Gd}^{3+}$ - dipicolinic acid derivatives as spin labels. Phys. Chem. Chem. Phys. 2011, 13, 10771-10780.

19. Manukovsky, N.; Frydman, V.; Goldfarb, D., Gd ${ }^{3+}$-spin labels report the conformation and solvent accessibility of solution and vesicle-bound melittin. J. Phys. Chem. B 2015, 119, 13732-13741.

20. Yulikov, M., Spectroscopically orthogonal spin labels and distance measurements in biomolecules. In Electron Paramagnetic Resonance, Gilbert, B. C.; Chechik, V.; Murphy, D. M., Eds. 2015; Vol. 24, pp 1-31. 
21. Garbuio, L.; Bordignon, E.; Brooks, E. K.; Hubbell, W. L.; Jeschke, G.; Yulikov, M., Orthogonal spin labeling and Gd(III)-nitroxide distance measurements on bacteriophage T4-lysozyme. J. Phys. Chem. B 2013, 117, 3145-3153.

22. Kaminker, I.; Tkach, I.; Manukovsky, N.; Huber, T.; Yagi, H.; Otting, G.; Bennati, M.; Goldfarb, D., W-band orientation selective DEER measurements on a $\mathrm{Gd}^{3+} /$ nitroxide mixed-labeled protein dimer with a dual mode cavity. $J$. Magn. Reson. 2013, 227, 66-71.

23. Kaminker, I.; Yagi, H.; Huber, T.; Feintuch, A.; Otting, G.; Goldfarb, D., Spectroscopic selection of distance measurements in a protein dimer with mixed nitroxide and $\mathrm{Gd}^{3+}$ spin labels. Phys. Chem. Chem. Phys. 2012, 14, 43554358.

24. Dalaloyan, A.; Qi, M.; Ruthstein, S.; Vega, S.; Godt, A.; Feintuch, A.; Goldfarb, D., Gd (III)-Gd (III) EPR distance measurements-the range of accessible distances and the impact of zero field splitting. Phys. Chem. Chem. Phys. 2015, 17, 18464-18476.

25. Manukovsky, N.; Feintuch, A.; Kuprov, I.; Goldfarb, D., Time domain simulation of $\mathrm{Gd}^{3+}-\mathrm{Gd}^{3+}$ distance measurements by EPR. J. Chem. Phys. 2017, 147, 044201.

26. Nitsche, C.; Otting, G., Pseudocontact shifts in biomolecular NMR using paramagnetic metal tags. Prog. Nucl. Magn. Reson. Spectrosc. 2017, 98-99, 20-49.

27. Martorana, A.; Bellapadrona, G.; Feintuch, A.; Di Gregorio, E.; Aime, S.; Goldfarb, D., Probing protein conformation in cells by EPR distance measurements using $\mathrm{Gd}^{3+}$ spin labeling J. Am. Chem. Soc. 2014, 136 13458-13465

28. Qi, M.; Gross, A.; Jeschke, G.; Godt, A.; Drescher, M., Gd(III)-PyMTA label is suitable for in-cell EPR. J. Am. Chem. Soc. 2014, 136, 15366-15378.

29. Yang, Y.; Yang, F.; Gong, Y. J.; Chen, J. L.; Goldfarb, D.; Su, X. C., A reactive, rigid Gd(III) labeling tag for In-cell EPR distance measurements in proteins. Angew. Chem. Int. Ed. 2017, 56, 2914-2918.

30. Raitsimring, A. M.; Astashkin, A. V.; Poluektov, O. G.; Caravan, P., High-field pulsed EPR and ENDOR of Gd ${ }^{3+}$ complexes in glassy solutions. Appl. Magn. Reson. 2005, 28, 281-295.

31. Raitsimring, A. M.; Gunanathan, C.; Potapov, A.; Efremenko, I.; Martin, J. M. L.; Milstein, D.; Goldfarb, D., Gd ${ }^{3+}$ complexes as potential spin labels for high field pulsed EPR distance measurements. J. Am. Chem. Soc. 2007, 129, 14138-14140.

32. Cohen, M. R.; Frydman, V.; Milko, P.; Iron, M. A.; Abdelkader, E. H.; Lee, M. D.; Swarbrick, J. D.; Raitsimring, A.; Otting, G.; Graham, B.; Feintuch, A.; Goldfarb, D., Overcoming artificial broadening in $\mathrm{Gd}^{3+}-\mathrm{Gd}^{3+}$ distance distributions arising from dipolar pseudo-secular terms in DEER experiments. Phys. Chem. Chem. Phys. 2016, 18, 12847-12859.

33. Collauto, A.; Frydman, V.; Lee, M. D.; Abdelkader, E. H.; Feintuch, A.; Swarbrick, J. D.; Graham, B.; Otting, G.; Goldfarb, D., RIDME distance measurements using Gd(III) tags with a narrow central transition. Phys. Chem. Chem. Phys. 2016, 18, 19037-19049.

34. Razzaghi, S.; Qi, M.; Nalepa, A. I.; Godt, A.; Jeschke, G.; Savitsky, A.; Yulikov, M., RIDME Spectroscopy with Gd(III) Centers. J. Phys. Chem. Lett. 2014, 5, 3970-3975.

35. Plato, M.; Steinhoff, H. J.; Wegener, C.; Torring, J. T.; Savitsky, A.; Mobius, K., Molecular orbital study of polarity and hydrogen bonding effects on the g and hyperfine tensors of site directed NO spin labelled bacteriorhodopsin. Mol. Phys. 2002, 100, 3711-3721.

36. Mobius, K.; Savitsky, A.; Wegener, C.; Plato, M.; Fuchs, M.; Schnegg, A.; Dubinskii, A. A.; Grishin, Y. A.; Grigor'ev, I. A.; Kuhn, M.; Duche, D.; Zimmermann, H.; Steinhoff, H. J., Combining high-field EPR with site-directed spin labeling reveals unique information on proteins in action. Magn. Reson. Chem. 2005, 43 Spec no., S4-s19.

37. Carmieli, R.; Papo, N.; Zimmermann, H.; Potapov, A.; Shai, Y.; Goldfarb, D., Utilizing ESEEM spectroscopy to locate the position of specific regions of membrane-active peptides within model membranes. Biophys. J. 2006, 90, 492-505.

38. Volkov, A.; Dockter, C.; Bund, T.; Paulsen, H.; Jeschke, G., Pulsed EPR determination of water accessibility to spinlabeled amino acid residues in LHCIIb. Biophys. J. 2009, 96, 1124-1141.

39. Lee, M. D.; Loh, C. T.; Shin, J.; Chhabra, S.; Dennis, M. L.; Otting, G.; Swarbrick, J. D.; Graham, B., Compact, hydrophilic, lanthanide-binding tags for paramagnetic NMR spectroscopy. Chem. Sci. 2015, 6, 2614-2624.

40. Loh, C. T.; Ozawa, K.; Tuck, K. L.; Barlow, N.; Huber, T.; Otting, G.; Graham, B., Lanthanide tags for site-specific ligation to an unnatural amino acid and generation of pseudocontact shifts in proteins. Bioconjugate Chem. 2013, 24, 260-268.

41. Graham, B.; Loh, C. T.; Swarbrick, J. D.; Ung, P.; Shin, J.; Yagi, H.; Jia, X.; Chhabra, S.; Barlow, N.; Pintacuda, G.; Huber, T.; Otting, G., DOTA-amide lanthanide tag for reliable generation of pseudocontact shifts in protein NMR spectra. Bioconjugate Chem. 2011, 22, 2118-2125.

42. Huang, C. H.; Morrow, J. R., A PARACEST agent responsive to inner- and outer-sphere phosphate ester interactions for MRI applications. J. Am. Chem. Soc. 2009, 131, 4206-4207. 
43. Barak, N. N.; Neumann, P.; Sevvana, M.; Schutkowski, M.; Naumann, K.; Malesevic, M.; Reichardt, H.; Fischer, G.; Stubbs, M. T.; Ferrari, D. M., Crystal structure and functional analysis of the protein disulfide isomerase-related protein ERp29. J. Mol. Biol. 2009, 385, 1630-1642.

44. Liepinsh, E.; Baryshev, M.; Sharipo, A.; Ingelman-Sundberg, M.; Otting, G.; Mkrtchian, S., Thioredoxin fold as homodimerization module in the putative chaperone ERp29: NMR structures of the domains and experimental model of the $51 \mathrm{kDa}$ dimer. Structure 2001, 9, 457-471.

45. Neylon, C.; Brown, S. E.; Kralicek, A. V.; Miles, C. S.; Love, C. A.; Dixon, N. E., Interaction of the Escherichia coli replication terminator protein (Tus) with DNA: a model derived from DNA-binding studies of mutant proteins by surface plasmon resonance. Biochemistry 2000, 39, 11989-11999.

46. Ozawa, K.; Headlam, M. J.; Schaeffer, P. M.; Henderson, B. R.; Dixon, N. E.; Otting, G., Optimization of an Escherichia coli system for cell-free synthesis of selectively N-labelled proteins for rapid analysis by NMR spectroscopy. Eur. J. Biochem. 2004, 271, 4084-4093.

47. Apponyi, M. A.; Ozawa, K.; Dixon, N. E.; Otting, G., Cell-free protein synthesis for analysis by NMR spectroscopy. Methods in molecular biology (Clifton, N.J.) 2008, 426, 257-268.

48. Goldfarb, D.; Lipkin, Y.; Potapov, A.; Gorodetsky, Y.; Epel, B.; Raitsimring, A. M.; Radoul, M.; Kaminker, I., HYSCORE and DEER with an upgraded 95 GHz pulse EPR spectrometer. J. Magn. Reson. 2008, 194, 8-15.

49. Mentink-Vigier, F.; Collauto, A.; Feintuch, A.; Kaminker, I.; Tarle, V.; Goldfarb, D., Increasing sensitivity of pulse EPR experiments using echo train detection schemes. J. Magn. Reson. 2013, 236, 117-125.

50. Clayton, J. A.; Qi, M.; Godt, A.; Goldfarb, D.; Han, S.; Sherwin, M. S., Gd $\mathrm{Gd}^{3+}-\mathrm{Gd}^{3+}$ distances exceeding $3 \mathrm{~nm}$ determined by very high frequency continuous wave electron paramagnetic resonance. Phys. Chem. Chem. Phys. 2017, 19, 5127-5136.

51. Edwards, D. T.; Ma, Z.; Meade, T. J.; Goldfarb, D.; Han, S.; Sherwin, M. S., Extending the distance range accessed with continuous wave EPR with $\mathrm{Gd}^{3+}$ spin probes at high magnetic fields. Phys. Chem. Chem. Phys. 2013, 15, 1131311326.

52. Takahashi, S.; Brunel, L. C.; Edwards, D. T.; van Tol, J.; Ramian, G.; Han, S.; Sherwin, M. S., Pulsed electron paramagnetic resonance spectroscopy powered by a free-electron laser. Nature 2012, 489, 409-413.

53. Benmelouka, M.; Van Tol, J.; Borel, A.; Port, M.; Helm, L.; Brunel, L. C.; Merbach, A. E., A high-frequency EPR study of frozen solutions of Gd(III) complexes: straightforward determination of the zero-field splitting parameters and simulation of the NMRD profiles. J. Am. Chem. Soc. 2006, 128, 7807-7816.

54. Epel, B.; Arieli, D.; Baute, D.; Goldfarb, D., Improving W-band pulsed ENDOR sensitivity-random acquisition and pulsed special TRIPLE. J. Magn. Reson. 2003, 164, 78-83.

55. Pannier, M.; Veit, S.; Godt, A.; Jeschke, G.; Spiess, H. W., Dead-time free measurement of dipole-dipole interactions between electron spins. J. Magn. Reson. 2000, 142, 331-340.

56. Jeschke, G.; Chechik, V.; Ionita, P.; Godt, A.; Zimmermann, H.; Banham, J.; Timmel, C. R.; Hilger, D.; Jung, H., DeerAnalysis2006 - a comprehensive software package for analyzing pulsed ELDOR data. Appl. Magn. Reson. 2006, 30, 473-498.

57. Stoll, S.; Schweiger, A., EasySpin, a comprehensive software package for spectral simulation and analysis in EPR. $J$. Magn. Reson. 2006, 178, 42-55.

58. Collauto, A.; Feintuch, A.; Qi, M.; Godt, A.; Meade, T.; Goldfarb, D., Gd(III) complexes as paramagnetic tags: Evaluation of the spin delocalization over the nuclei of the ligand. J. Magn. Reson. 2016, 263, 156-163.

59. Schweiger, A.; Jeschke, G., Principles of pulse electron paramangetic resonance. Oxford University Press: 2001.

60. Kolhe, P.; Amend, E.; Singh, S. K., Impact of freezing on $\mathrm{pH}$ of buffered solutions and consequences for monoclonal antibody aggregation. Biotechnol Prog 2010, 26, 727-733.

61. Garbuio, L.; Zimmermann, K.; Haussinger, D.; Yulikov, M., Gd(III) complexes for electron-electron dipolar spectroscopy: Effects of deuteration, $\mathrm{pH}$ and zero field splitting. J. Magn. Reson. 2015, 259, 163-173.

62. Epel, B.; Manikandan, P.; Kroneck, P. M. H.; Goldfarb, D., High-field ENDOR and the sign of the hyperfine coupling. Appl. Magn. Reson. 2001, 21, 287-297.

63. Caravan, P.; Astashkin, A. V.; Raitsimring, A. M., The gadolinium(III)-water hydrogen distance in MRI contrast agents. Inorg. Chem. 2003, 42, 3972-3974.

64. Chin, K. O. A.; Morrow, J. R.; Lake, C. H.; Churchill, M. R., Synthesis and solution properties of Lanthanum(IIi), Europium(III), and Lutetium(III) THP complexes and an X-ray diffraction study of a crystal containing 4 sereoisomers of a Europium(III) THP complex (THP = 1,4,7,10-tetrakis(2-hydroxypropyl)-1,4,7,10-tetraazacyclododecane) - Methylgroups impart rigidity to S,S,S,S-THP macrocyclic complexes. Inorg. Chem. 1994, 33, 656-664.

65. Khan, S.; Peters, V.; Kowalewski, J.; Odelius, M., Zero-field splitting in the isoelectronic aqueous Gd(III) and Eu(II) complexes from a first principles analysis. Chem. Phys. 2018, 503, 56-64. 


\section{TOC graphics + synopsis}

The $\mathbf{C 7}$ and $\mathbf{C 8}$ tags are compact hydrophilic cyclen-based Gd(III) tags for conjugation to cysteine residues in proteins that can be used in EPR based distance measurements for tracking proteins' conformations. They produce narrow distance distributions and, more uniquely, their EPR spectra are sensitive to the labeling position, reporting on environmentdependent charge density on the Gd(III)-coordinating oxygens, possibly owing to different degrees of H-bonding and interaction with anions.

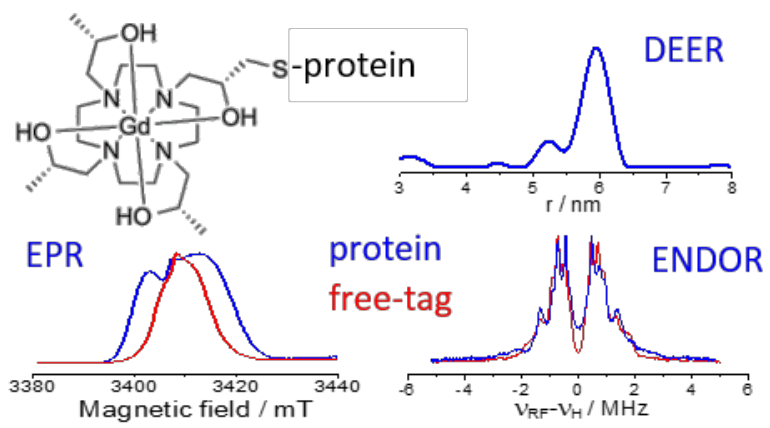




\section{Supplementary information}

\section{Small Gd(III) Tags for Gd(III)-Gd(III) Distance Measurements in Proteins by EPR Spectroscopy}

G. Prokopiou ${ }^{1}$, M. D. Lee ${ }^{2}$, A. Collauto ${ }^{1}$, E. H. Abdelkader ${ }^{3}$, T. Bahrenberg ${ }^{1}$, A. Feintuch ${ }^{1}$, M. RamirezCohen $^{1}$, J. Clayton ${ }^{4}$, J. D. Swarbrick², B. Graham², G. Otting ${ }^{3}$, D. Goldfarb ${ }^{1}$

${ }^{1}$ Department of Chemical Physics, Weizmann Institute of Science, Rehovot, 76100, Israel

${ }^{2}$ Monash Institute of Pharmaceutical Sciences, Monash University, Parkville, VIC 3052, Australia

${ }^{3}$ Research School of Chemistry, Australian National University, Canberra, ACT 2601, Australia

${ }^{4}$ Department of Physics, University of California, Santa-Barbara, CA 93106-9530, USA

\section{Contents}

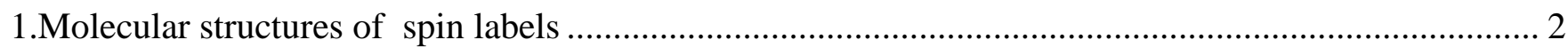

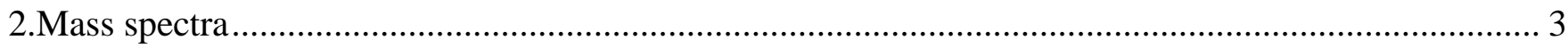

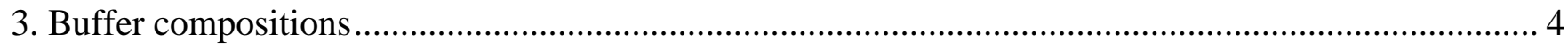

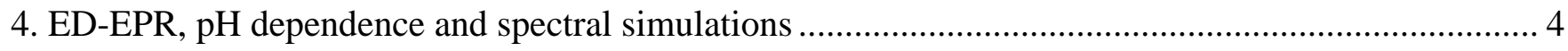

5. pH dependence of the ED-EPR spectra of ERp29-S114C-C8.......................................................... 6

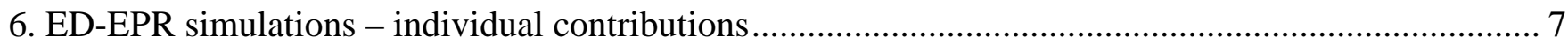

7. Comparison of ENDOR spectra................................................................................................. 7

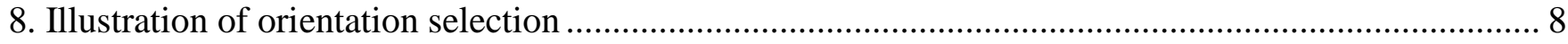

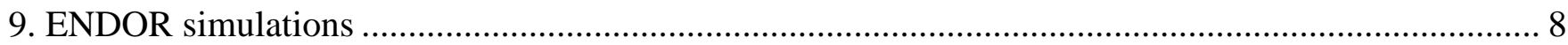

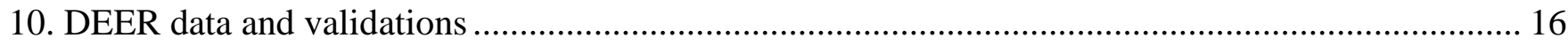

11. Simulations of ED-EPR spectra of other tags ................................................................................. 17 


\section{Molecular structures of spin labels}
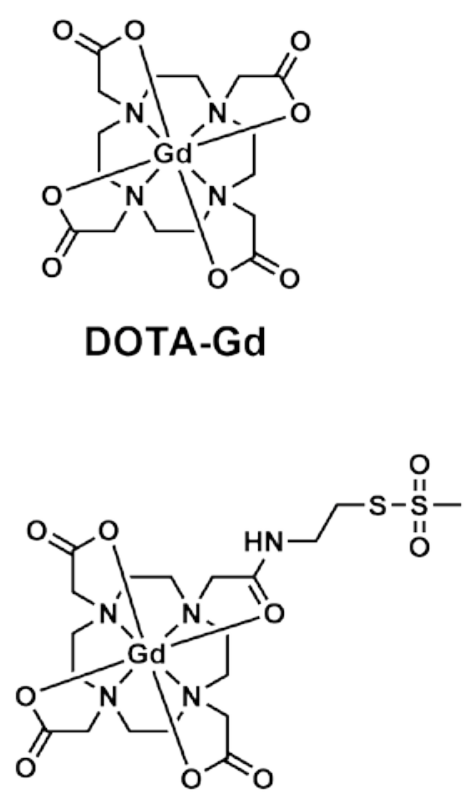

MTS-ADO3A-Gd

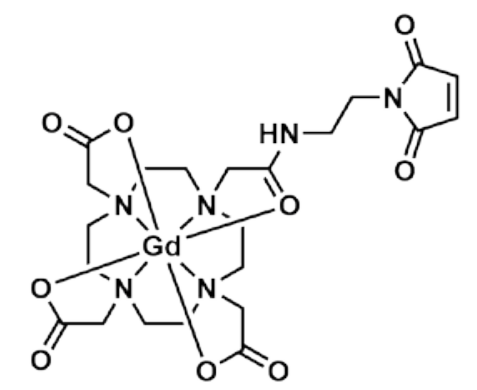

Maleimide-DO3A-Gd

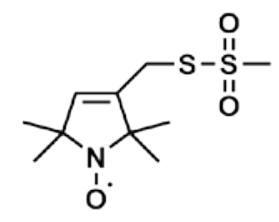

MTSL

Figure S1. Chemical structures of MTSL and Gd(III) complexes referred to in the text. 


\section{Mass spectra}
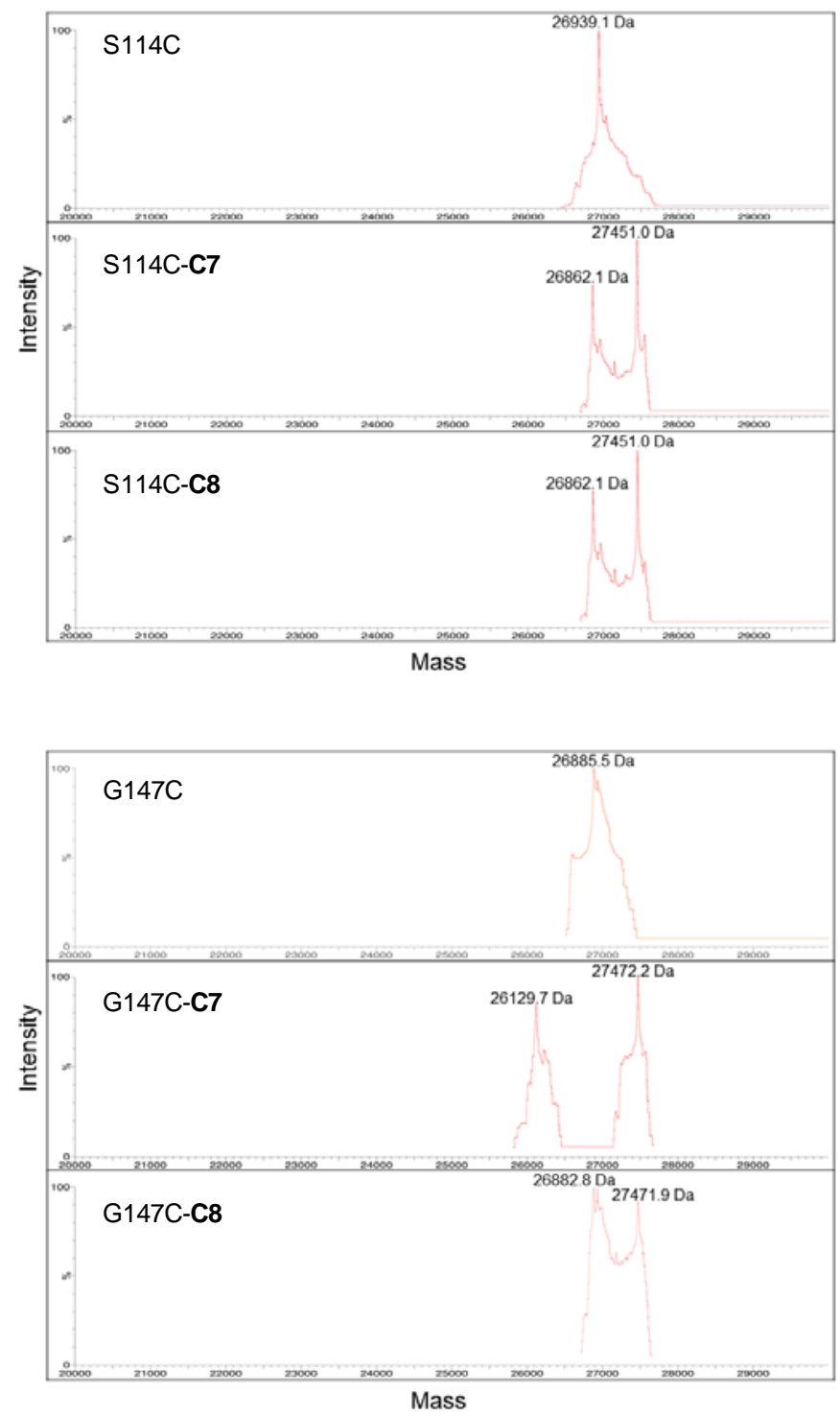

Figure S2. Mass spectrometric analysis of labeling yields. The spectra were recorded with $0.1 \%$ formic acid on a Waters LCT mass spectrometer. (A) The mass of unlabeled ERp29 S114C corresponds to the adduct with $\beta$-mercaptoethanol (expected mass: $26938 \mathrm{Da}$ ). The adduct arose from long-term storage in the presence of $\beta$-mercaptoethanol. Following ligation with the $\mathbf{C 7}$ or $\mathbf{C 8}$ tags, the mass difference between unreacted protein $(26862 \mathrm{Da})$ and tagged protein $(27451 \mathrm{Da})$ by $589 \mathrm{Da}$ is consistent with the expected mass increase (592 Da). (B) The mass of unlabeled ERp29 G147C is close to the expected mass (26886 Da). Following ligation with the $\mathbf{C} 7$ or $\mathbf{C 8}$ tags, the mass peaks at $27472 \mathrm{Da}$ correspond to an increase by $586 \mathrm{Da}$ relative to the unligated protein (expected: $592 \mathrm{Da}$ ). The peak corresponding to the unlabeled G147C-C7 has a lower mass than expected, which we attribute to degradation during storage. It is inconsequential as only the labeled protein was detected by the EPR measurements. 


\section{Buffer compositions}

Table S1. Buffer Compositions for pH Dependent Measurements on the Free C7/C8 Tags

\begin{tabular}{lll}
\hline $\mathrm{pH}$ & $0.2 \mathrm{M} \mathrm{Na}_{2} \mathrm{HPO}_{4} / \mu \mathrm{L}$ & $0.1 \mathrm{M}$ citric acid$/ \mu \mathrm{L}$ \\
\hline 3.0 & & \\
4.0 & 20.5 & 79.5 \\
5.0 & 37.0 & 63.0 \\
6.0 & 49.3 & 50.7 \\
7.0 & 62.1 & 37.9 \\
8.0 & 82.3 & 17.7 \\
9.0 & 95.8 & 4.20 \\
\hline
\end{tabular}

a The buffers were prepared in $\mathrm{H}_{2} \mathrm{O}$.

\section{ED-EPR, pH dependence and spectral simulations}

a)

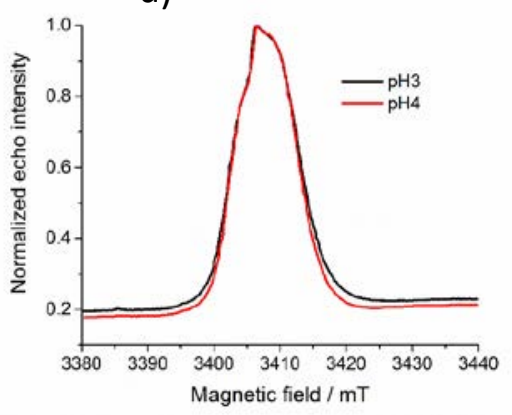

c)

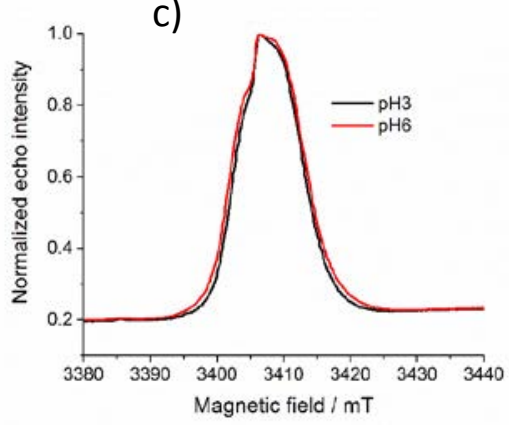

b)

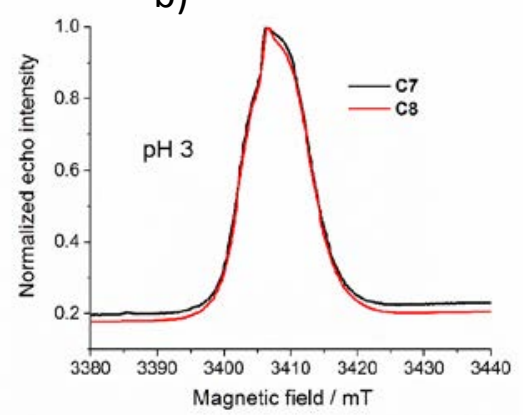

d)

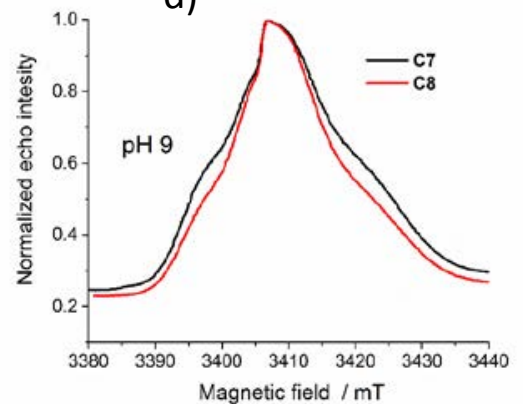

Figure S3. Central transition region of ED-EPR spectra. (a,c) Comparison of $\mathbf{C 7}$ at different pH. (b,d) Comparison of $\mathbf{C 7}$ and $\mathbf{C 8}$ at different pH values. The buffers used are listed in Table S1. 

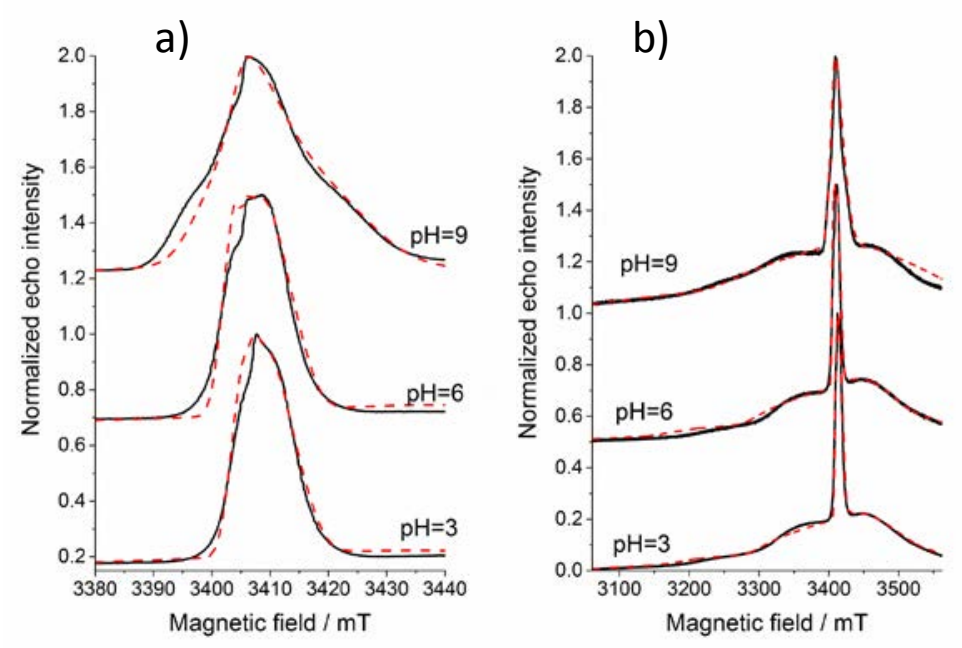

Figure S4. ED-EPR spectra. (a) Central transition region and (b) full range of the spectra of the $\mathbf{C 8}$ tag at different $\mathrm{pH}$ along with simulations (red dashed lines) performed with the parameters listed in Table S3. Spectra recorded at $\mathrm{pH}=8$ were similar to those of $\mathrm{pH}=6$ (not shown).
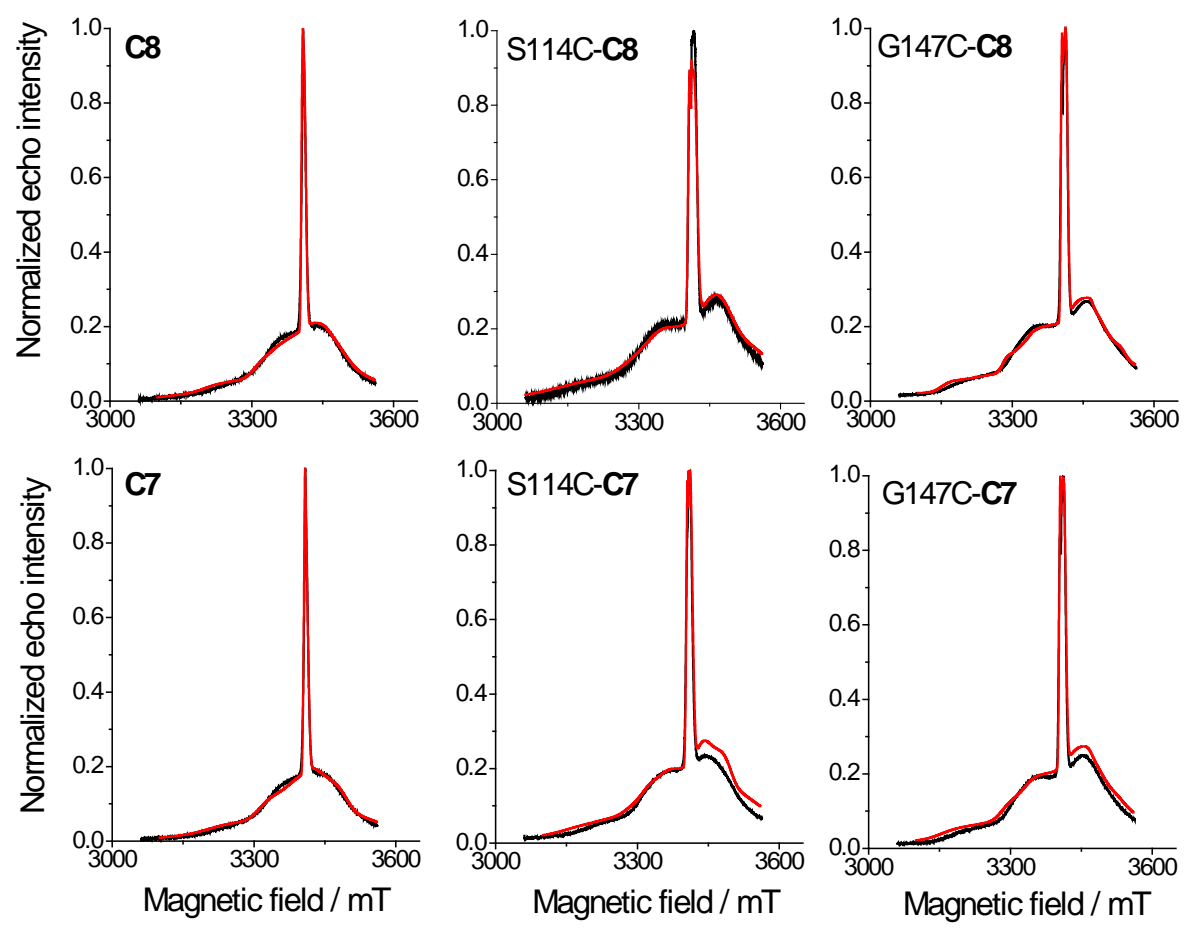

Figure S5. W-band ED-EPR spectra of the samples indicated on the figure and described in Figure 3 in the main text. The experimental and simulated spectra are shown in black and red, respectively. The simulation parameters are given in Table 1. 
Table S2. Parameters Used for Simulating the Spectra of the C7 tag (not shown).

\begin{tabular}{cccccc}
\hline $\mathbf{p H}$ & $\boldsymbol{g}$ & $\boldsymbol{D}, \mathbf{M H z}$ & $\boldsymbol{E} / \mathbf{D}$ & $\begin{array}{c}\text { D strain, } \\
\mathbf{M H z}\end{array}$ & $\begin{array}{c}\boldsymbol{E} \text { strain, } \\
\mathbf{M H z}\end{array}$ \\
\hline & & & & & \\
6 & 1.9907 & -1650 & 0.18 & 450 & 300 \\
9 & 1.9907 & -1690 & 0.18 & 450 & 300 \\
9 & 1.9902 & -2300 & 0.20 & 950 & 650 \\
\hline
\end{tabular}

Table S3. Parameters Used for Simulating the Spectra of the C8 Tag, Shown in Figure S4.

\begin{tabular}{cccccc}
\hline $\mathbf{p H}$ & $\boldsymbol{g}$ & $\boldsymbol{D}, \mathbf{M H z}$ & $\boldsymbol{E} / \mathbf{D}$ & $\begin{array}{c}\text { D strain, } \\
\mathbf{M H z}\end{array}$ & $\begin{array}{c}\boldsymbol{E} \text { strain, } \\
\mathbf{M H z}\end{array}$ \\
\hline & & & & & \\
6 & 1.9900 & -1600 & 0.200 & 450 & 350 \\
9 & 1.9907 & -1650 & 0.18 & 450 & 300 \\
9 & 1.9904 & -2240 & 0.27 & 850 & 400 \\
\hline
\end{tabular}

5. pH dependence of the ED-EPR spectra of ERp29-S114C-C8

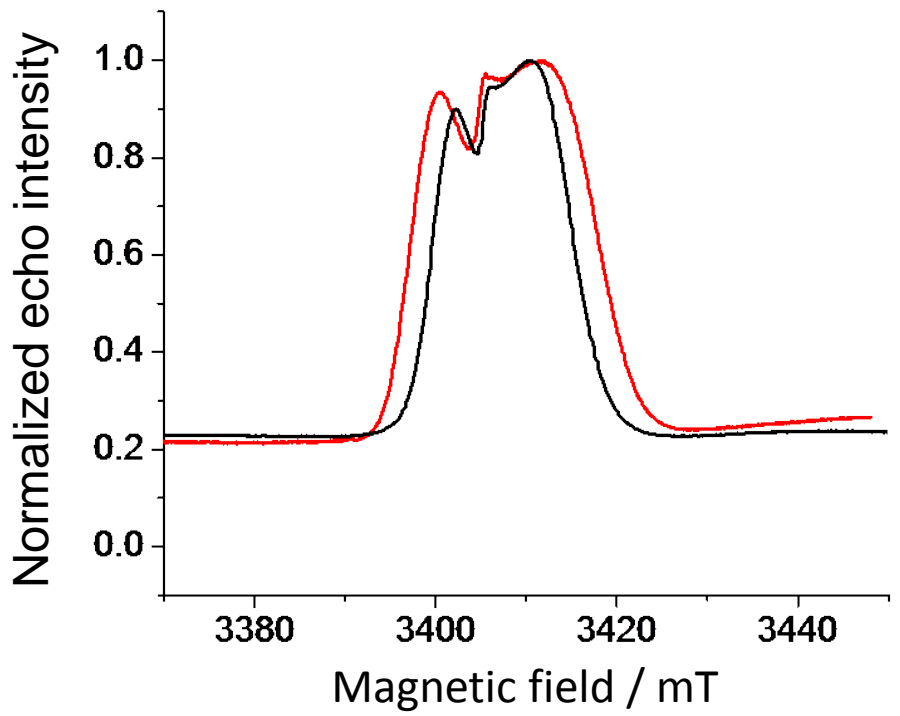

Figure S6. Central transition region of W-band ED-EPR spectra of S114C-C8 tag, at pH = 8 (black, using Tris- $\mathrm{HCl}$ buffer to adjust the $\mathrm{pH}$ ) and $\mathrm{pH}=4.9$ (red, using MES buffer to adjust the $\mathrm{pH}$ ). 


\section{ED-EPR simulations - individual contributions}
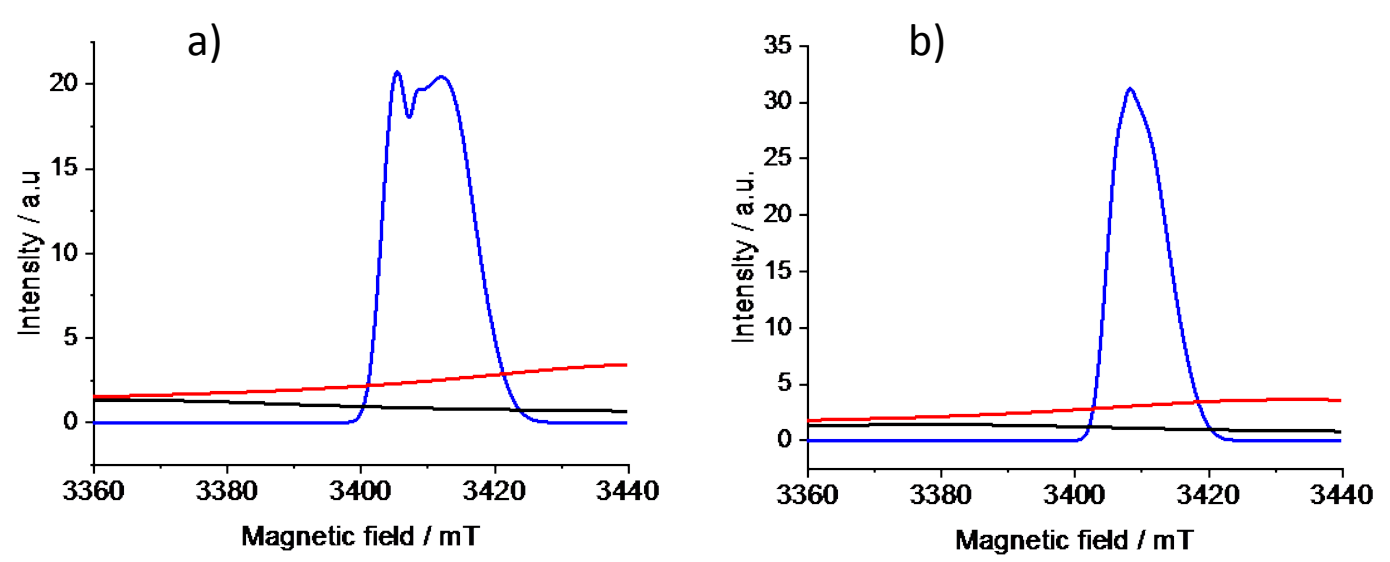

Figure S7. Contributions of central transition (blue), the $|-3 / 2>\rightarrow|-1 / 2>$ transition (red), and the $\mid 1 / 2$ $>\rightarrow \mid 3 / 2>$ transition (black) to the simulated spectra of (a) G147C-C8 and (b) free $\mathbf{C 8}$ tag (no buffer).

\section{Comparison of ENDOR spectra}

(a)

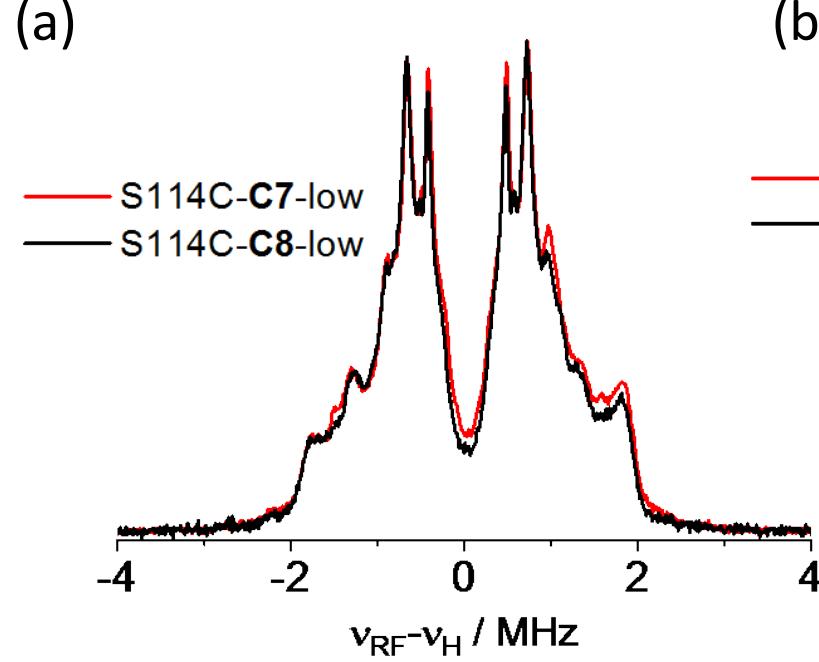

(b)

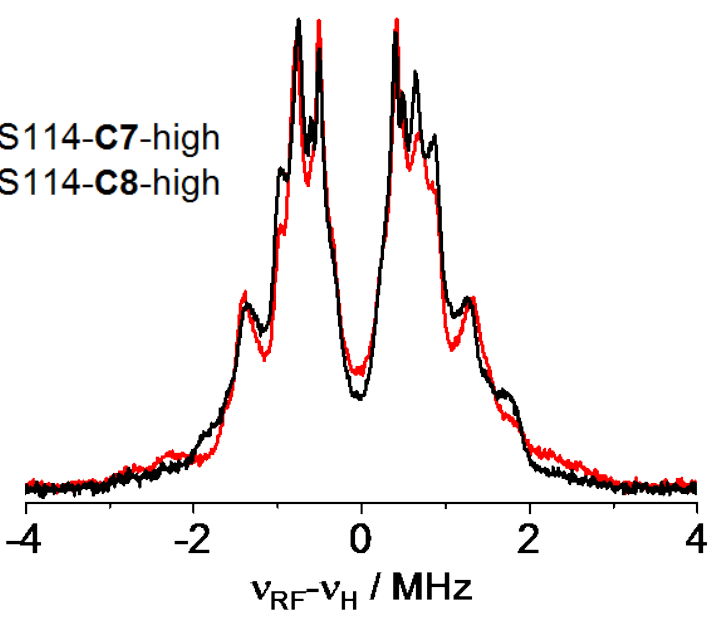

Figure S8. Comparison of the Davies ${ }^{1} \mathrm{H}$-ENDOR spectra of S114C-C8 and S114C-C7 recorded at the (a) low- and (b) high-field maxima. 


\section{Illustration of orientation selection}

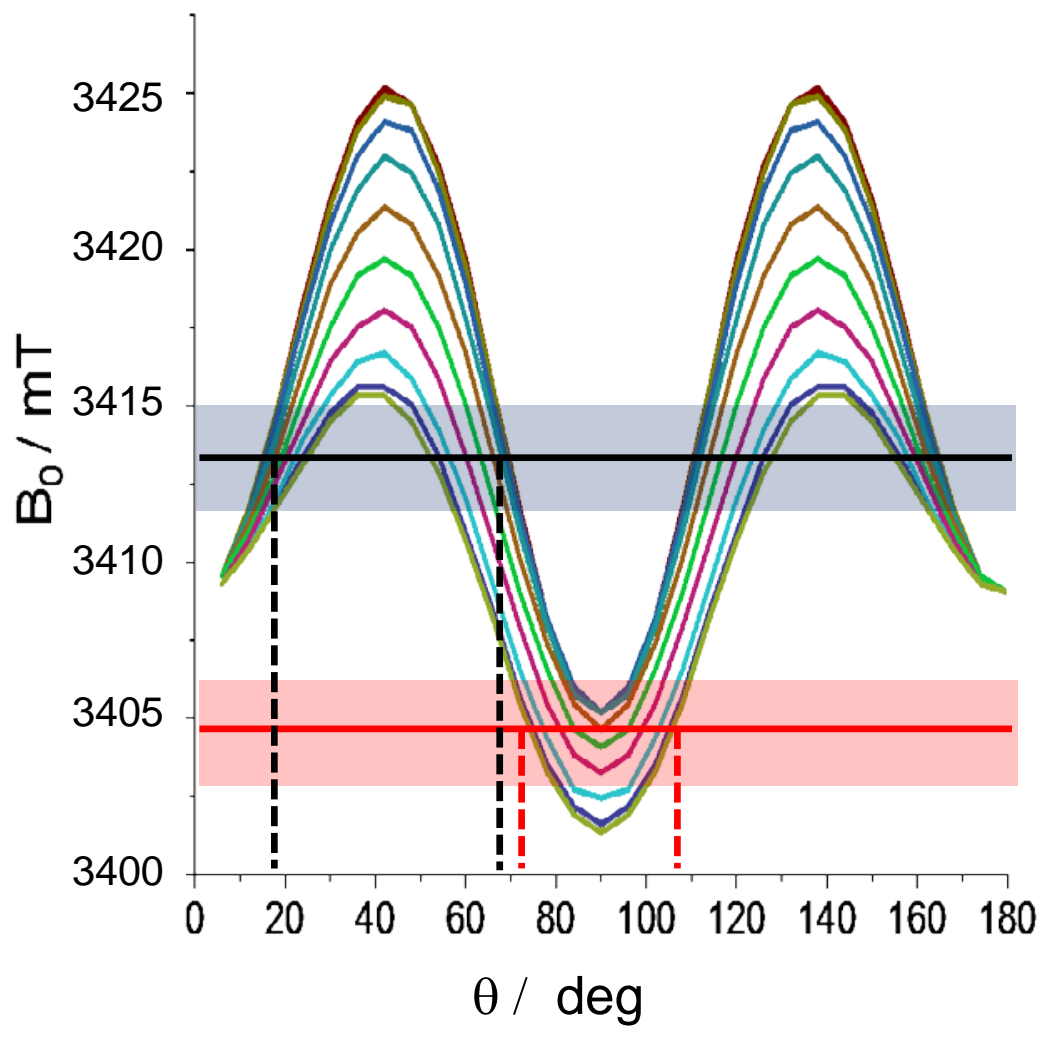

Figure S9. Illustration of the orientation selection for S114C-C8. The curves represent the EPR peak position calculated for the $D$ and $E$ values and the corresponding strains (see Table 1 , bottom values) as a function of the polar angle $\theta$ of the principal axis system of the ZFS with respect to the magnetic field direction for a range of azimuthal angles $\phi$ in the range of $0-180^{\circ}$, in steps of $10^{\circ}$. The black and red horizontal lines correspond to the high- and low-field positions at which the respective ENDOR spectra were recorded. The vertical dotted lines show the corresponding ranges of orientations selected. The transparent black and red boxes represent the $100 \mathrm{MHz}$ excitation width used for selecting the orientations.

\section{ENDOR simulations}

Figure S10a shows the distributions of the Eu-H distances obtained from the (S)-THP-Eu(III) crystal structure. $^{1}$ Like the DOTA-Gd(III) complex, the (S)-THP-Eu(III) complex also contains two groups of hydrogens at distances grouped in the region of 3.4-3.8 and 4.1-4.6 $\AA$, but there are also hydrogens at 4.9$5.4 \AA$ from the metal ion that are assigned to the methyl protons. A simulation using these distances, compared to the experimental spectrum of the $\mathbf{C 8}$ tag (no buffer), is shown in Figure S10b. The fit is not satisfactory as the signals at about $\pm 2 \mathrm{MHz}$ are missing. To include potential contributions arising from 
the $\pm 3 / 2$ electron spin manifolds, we repeated the simulation for an $S=3 / 2$ system. The simulations assumed room temperature and therefore showed no asymmetry due to the higher population of the $|-3 / 2\rangle$ $\rightarrow|-1 / 2\rangle$ transition as compared to the $|1 / 2\rangle \rightarrow|3 / 2\rangle$ transition expected at $10 \mathrm{~K}$. These simulations (Figure S10c) led to a better agreement of the peak positions but the doublet at $\pm 0.5 \mathrm{MHz}$ is not reproduced and the contributions of the large couplings are exaggerated.

a)

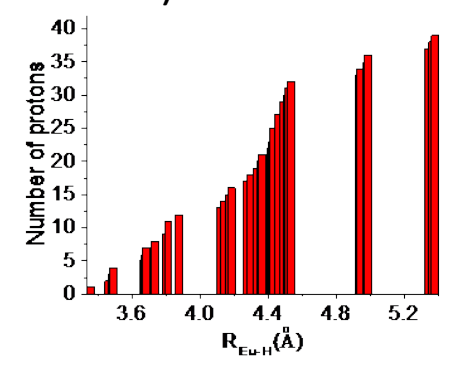

b)

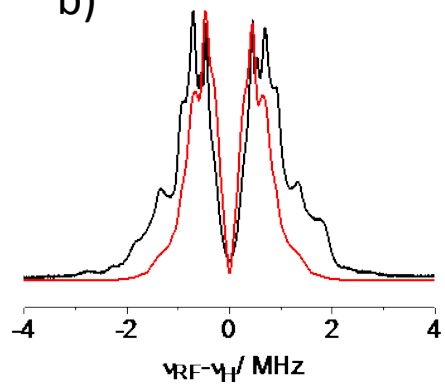

c)

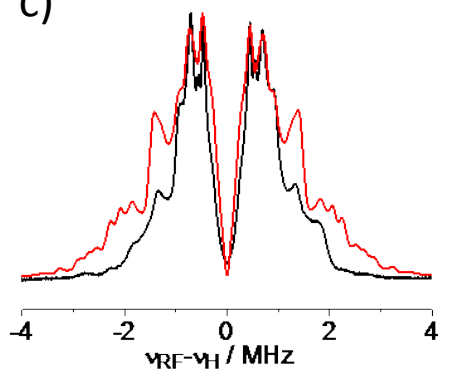

Figure S10. Simulation of ${ }^{1} \mathrm{H}$-ENDOR spectra. (a) Eu-H distances of the various protons in the crystal structure of the $\mathrm{Eu}(\mathrm{III})$ complex of 1,4,7,10-tetrakis((S)- 2-hydroxypropyl)-1,4,7,10tetraazacyclododecane ((S)-THP), which is the 4-fold symmetric compound upon which the $\mathbf{C} 7$ tag is based. The large number of protons is a consequence of the number of molecules in the unit cell (16). (b) Comparison of the experimental (black) and simulated (red) spectrum of the $\mathbf{C 7}$ tag. The simulations took into account only the $\pm 1 / 2$ manifolds. (c) Same as (b), except that the simulated spectrum included the contributions from both $\pm 1 / 2$ and $\pm 3 / 2$ electron spin manifolds. See text for details.

In order to improve the fitting and remove the fine structure observed in the calculated spectrum of Figure S10c, the different hydrogens were grouped into four classes of non-exchangeable protons according to their couplings with $\mathrm{Gd}(\mathrm{III})$ (Figure 6a and Table 2). When orientation selection is taken into account the Euler angles relating the orientation of the Gd-H bond direction with respect to the ZFS principal axis should be specified. Considering that the dipolar interaction is axially symmetric while the ZFS is not, two of the three Euler angles should be non-zero. As we do not know the direction of the ZFS within the molecule, we set two of the angles to zero and used $(0, \beta, 0)$, where the same $\beta$ value was employed for all the protons in a particular class. In reality, we expect these to be distributed, just as $D$ and $E$ are. In the simulations we searched for the $\beta$ values which give the best agreement with the experiment. The bestfitting parameters given in Table 2 were obtained by manual adjustment. Figure S11 shows the individual contributions of the different protons simulated for G147C-C8. The simulations of all samples, considering contributions from the central transition only, are shown in Figure S12. While a good agreement was obtained for the high-field position, the agreement for the low-field spectrum is worse in 
terms of the relative intensities. This is particularly evident for the intensity and asymmetry of the peaks around $\pm 2 \mathrm{MHz}$ in the low-field spectrum. In these simulations, $\beta=40^{\circ}$ was taken for the protons of class $\mathrm{B}\left(T_{\perp}=1.45 \mathrm{MHz}\right)$. Setting $\beta=90^{\circ}$ for the $\mathrm{B}$ class protons improved the fit for the low-field spectra but deteriorated the fit for the high-field positions (Figure S13).

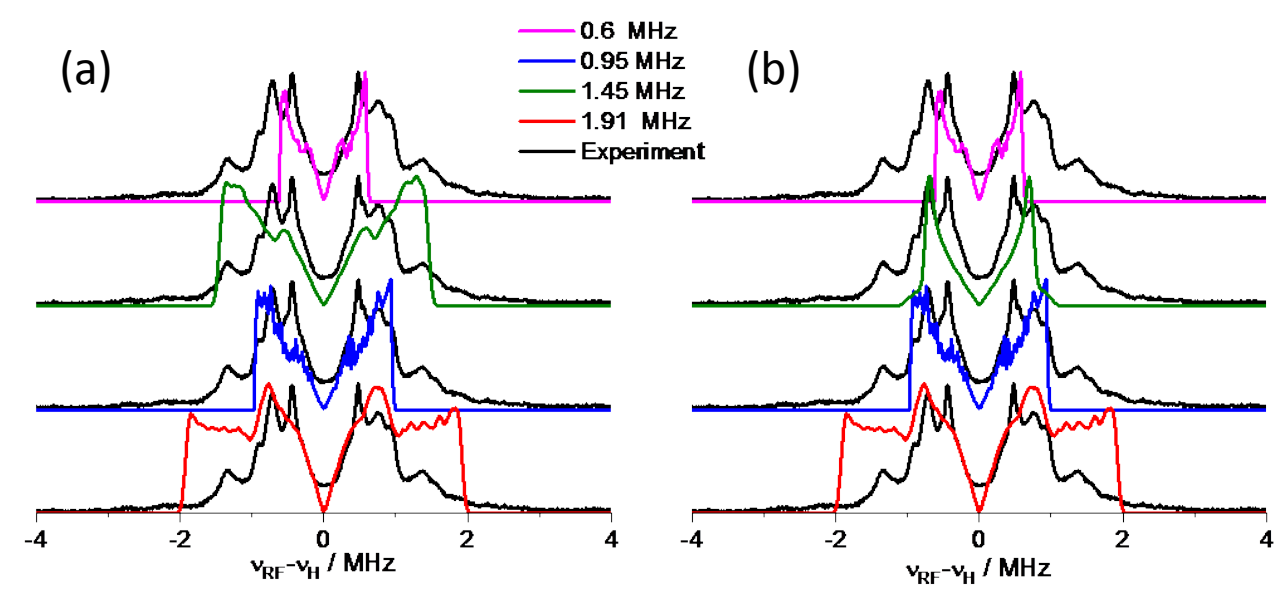

Figure S11. The individual contributions of simulated spectra of the protons with $0.6 \mathrm{MHz}$ (magenta), $1.45 \mathrm{MHz}$ (green), $0.95 \mathrm{MHz}$ (blue), and $1.91 \mathrm{MHz}$ (red) couplings for G147C-C8, recorded at the highfield position, as compared to the experimental spectrum (black). Only contributions from the central transition are considered. (a) $\beta=40^{\circ}$ and (b) $\beta=90^{\circ}$ for the proton with the $1.45 \mathrm{MHz}$ coupling (class B). 
a) G147C-C8 low

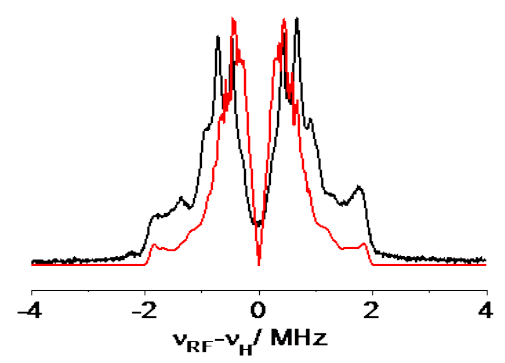

d) S114C-C8 low

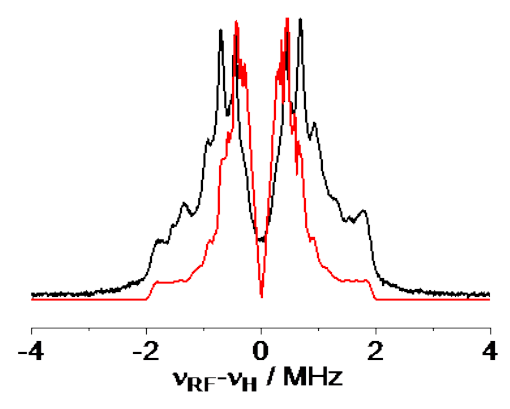

g) S114C-C7 high

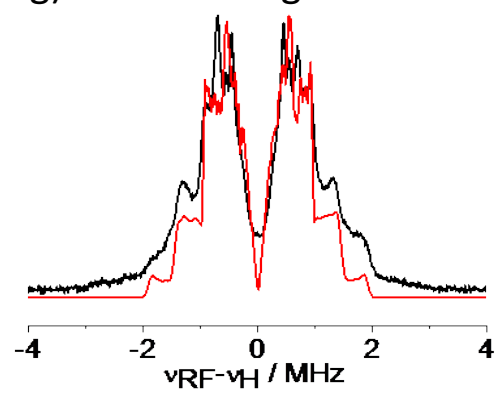

b'

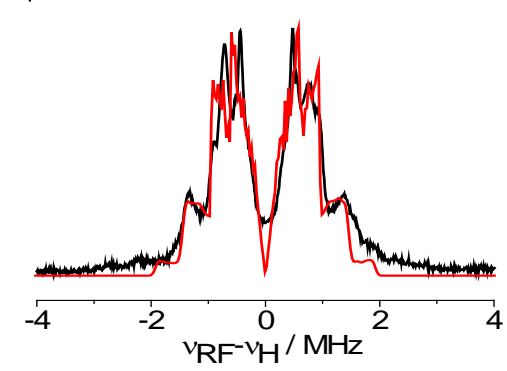

e)

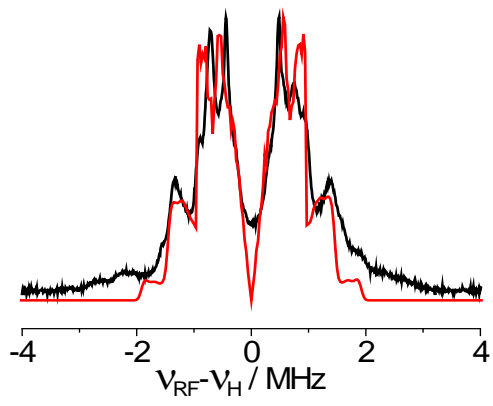

n.

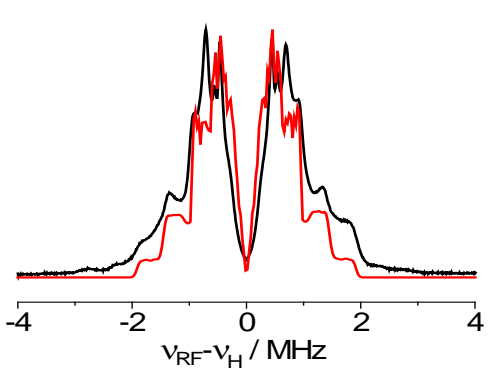

c) G147C-C7 low

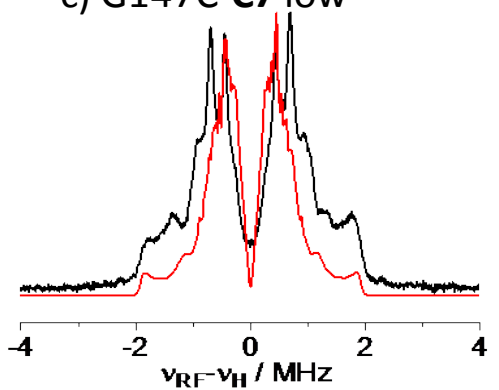

f) S114C-C7 low

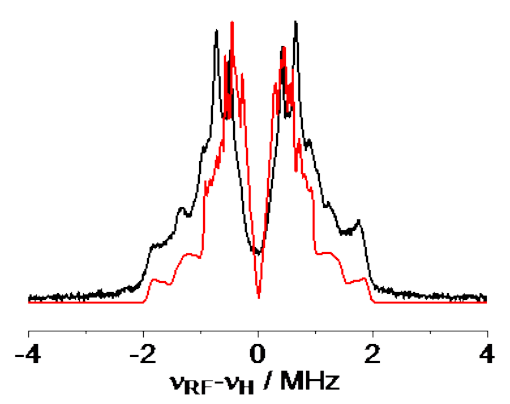

i) $\mathrm{C7}$

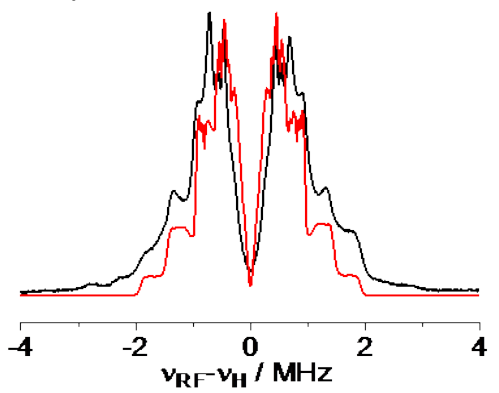

Figure S12. Experimental (black) and simulated (red) Davies ${ }^{1} \mathrm{H}$-ENDOR spectra of the samples noted on the figure. The simulations considered only the central transition and $\beta=40^{\circ}$ for class B protons. Differences between the simulated spectra arise from different orientation selections because their EPR spectra are different and not because of different hyperfine parameters. Spectra of the free tags were recorded without added buffer. 


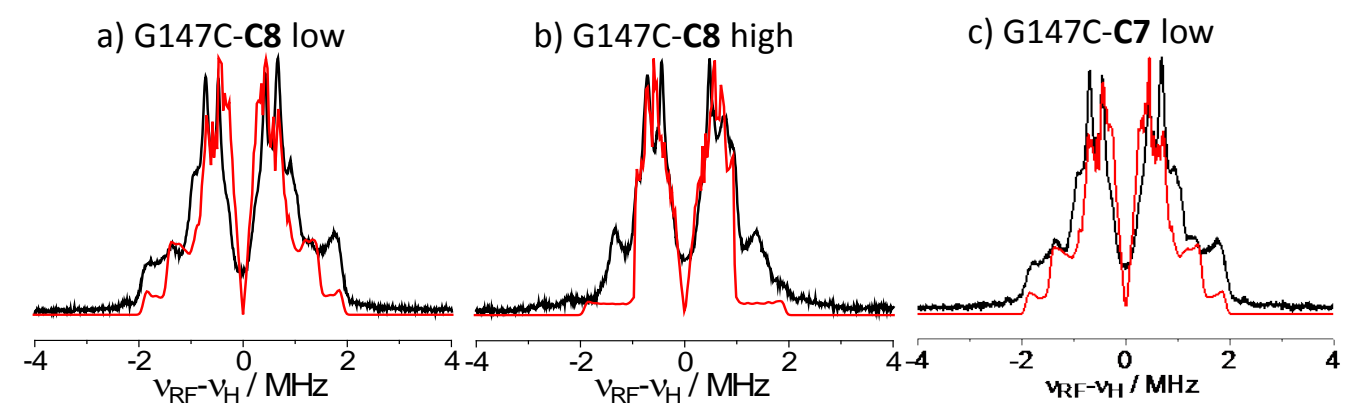

d) S114C-C8 low

e) S114C-C8 high
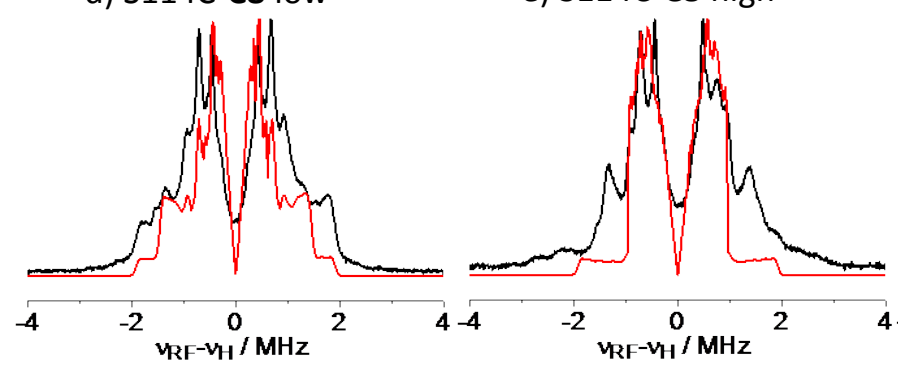

f) S114C-C7 low

g) S114C-C7 high

h) $\mathbf{C} 8$

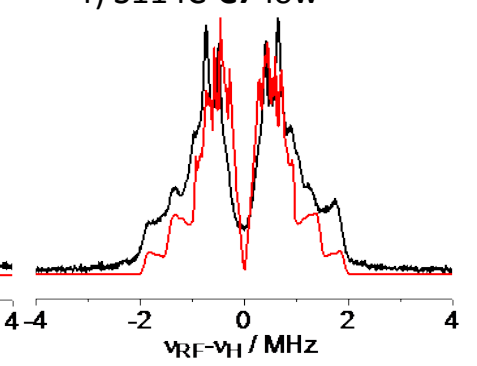

i) $\mathrm{C7}$

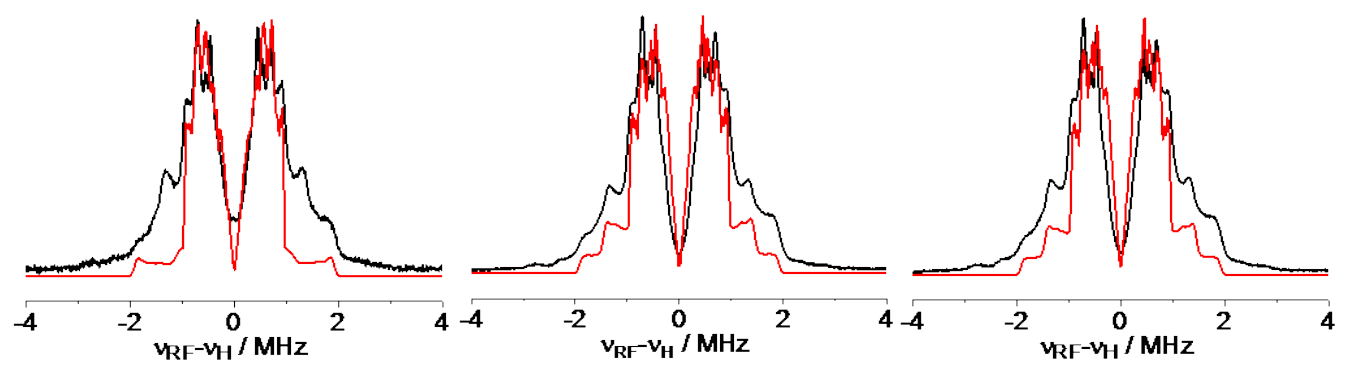

Figure S13. Same as Figure S12 but for $\beta=90^{\circ}$ for the class B protons.

As mentioned earlier, the asymmetry in the intensities of the doublets at $\pm 2 \mathrm{MHz}$ suggests contributions from manifolds other than the $\pm 1 / 2$ manifolds, mostly from the $-3 / 2$ manifold, but also from the $+3 / 2$ manifold. These contributions seem particularly crucial for the protons with the small couplings, namely classes $C$ and $D$. The signals associated with the $\pm 1 / 2$ manifolds are largely suppressed by the blind spot of the Davies ENDOR sequence in the center of the spectrum. The inclusion of these manifolds produces peaks around $\pm 2 \mathrm{MHz}$ as shown in Figure S14. The other protons with the larger couplings contribute broader, mostly unresolved powder patterns that are evident at the wings of the spectra. A better fit could be obtained for the free tags and the high-field spectra by including small contributions from these transitions (50\% and $12.5 \%$ for the $-3 / 2$ and $-3 / 2$ manifolds, respectively) for the class C- and D- protons, as shown in Figure S15 for $\beta=40^{\circ}$. For the low-field spectra the improvement was not significant. The effect of including the contributions of the $\pm 3 / 2$ manifolds for $\beta=90^{\circ}$ for the class B protons is shown in 
Figure S16. We prefer the $\beta=40^{\circ}$ set of simulations because of the better agreement it produces for the doublets with the large splittings.

(a)
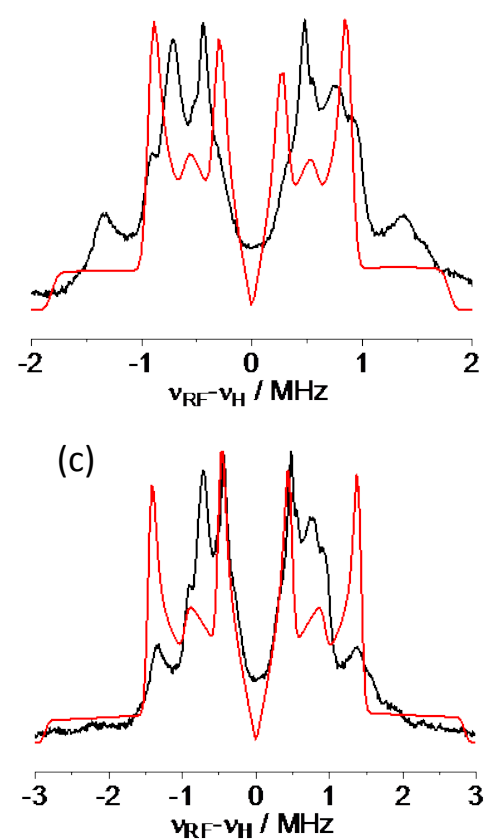

(b)

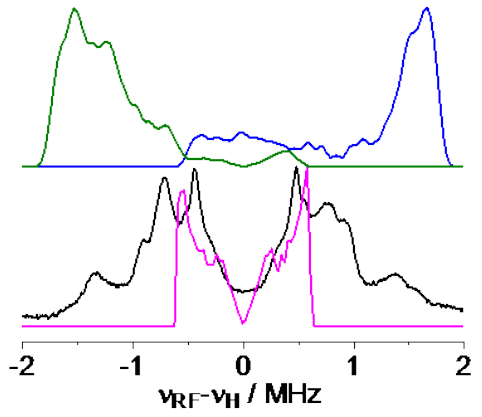

(d)

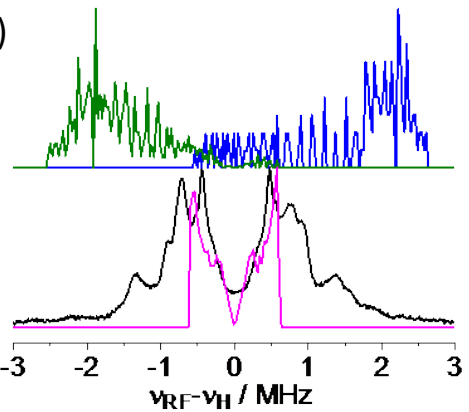

Figure S14. Davies ${ }^{1} \mathrm{H}$-ENDOR spectra of G147C-C8. (a) Experimental spectrum recorded at the high field position (black) and a simulation (red) assuming an $S=3 / 2$ spin system (without orientation selection) and $T_{\perp}=0.6 \mathrm{MHz}$. (b) The bottom panel shows a superimposition of the experimental spectrum (black) with a simulation of a $S=1 / 2$ system with a coupling of $0.6 \mathrm{MHz}$ including the orientation selection of the central EPR transition (magenta), which was derived from corresponding simulations of the EPR spectrum simulated for $S=7 / 2$. The top panel shows the simulated contributions of the $\pm 3 / 2$ manifolds according to their associated selected orientations as derived from the EPR spectral simulations assuming a $S=1 / 2$ system with a 3 -fold larger $T_{\perp}$ value of $1.8 \mathrm{MHz}$. The spectra corresponding to the $\mathrm{m}_{S}=-3 / 2$ (blue) and 3/2 (green) are displayed separately. (c) Same as (a) but for $T_{\perp}=0.95 \mathrm{MHz}$. (d) Same as (b) but for $T_{\perp}=2.85 \mathrm{MHz}$. 


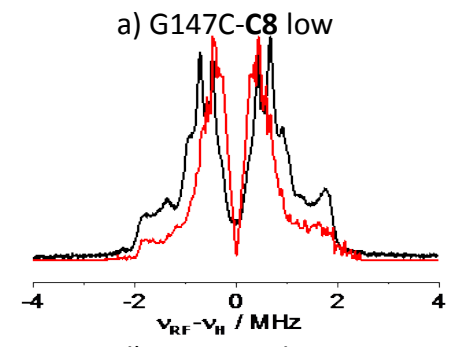

d) S114C-C8 low

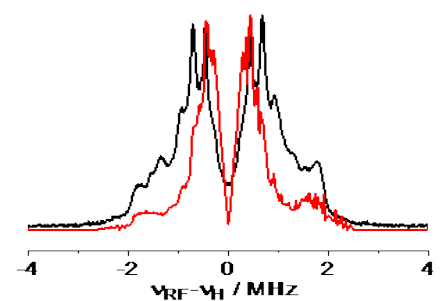

g) S114C-C7 high

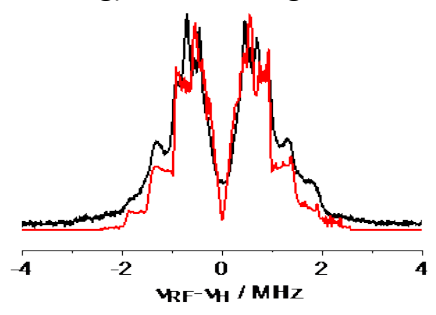

b) G147C-C8 high

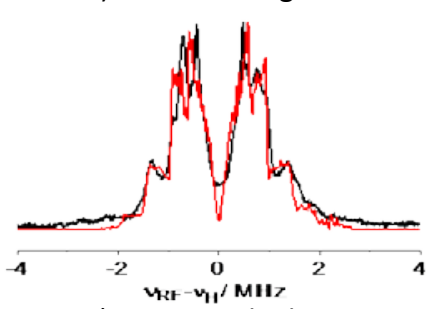

e) S114C-C8 high

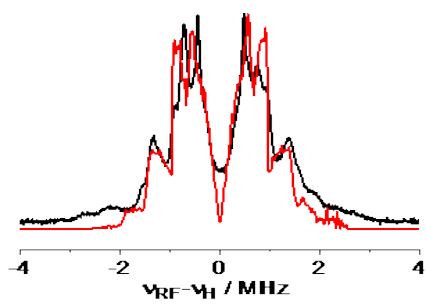

h) $\mathbf{C 8}$

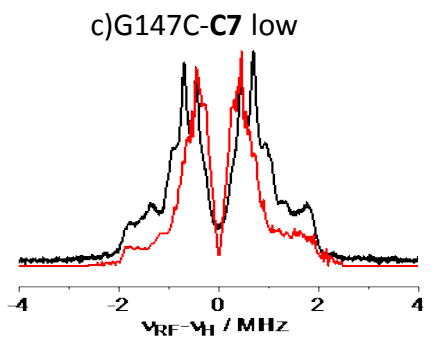

f) S114C-C7 low

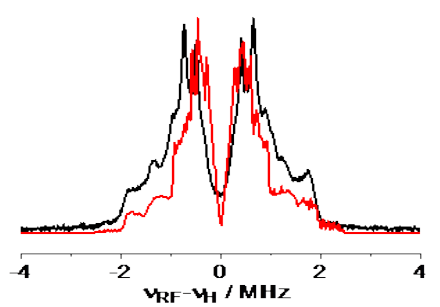

i) $\mathbf{C 7}$
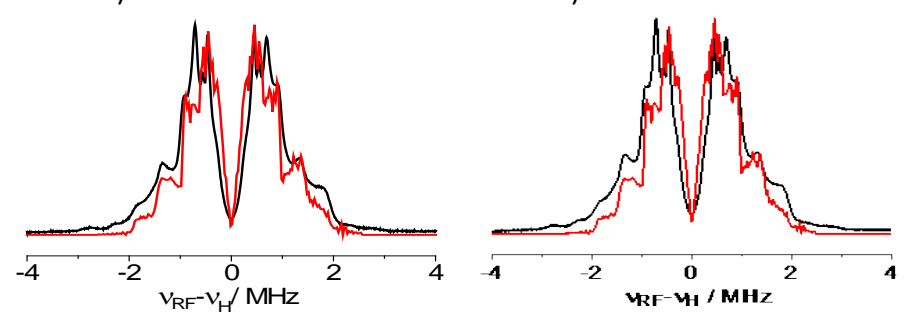

Figure S15. Experimental (black) and simulated (red) Davies ${ }^{1} \mathrm{H}$-ENDOR spectra with the inclusion of the contributions from the $\pm 3 / 2$ transitions (relative weights are $50 \%$ for the $-3 / 2$ manifold and $12.5 \%$ for the $+3 / 2$ manifold) only for class $\mathrm{C}$ and $\mathrm{D}$ protons and using $\beta=40^{\circ}$ for the class B protons. Spectra of the free tags were recorded without added buffer. 

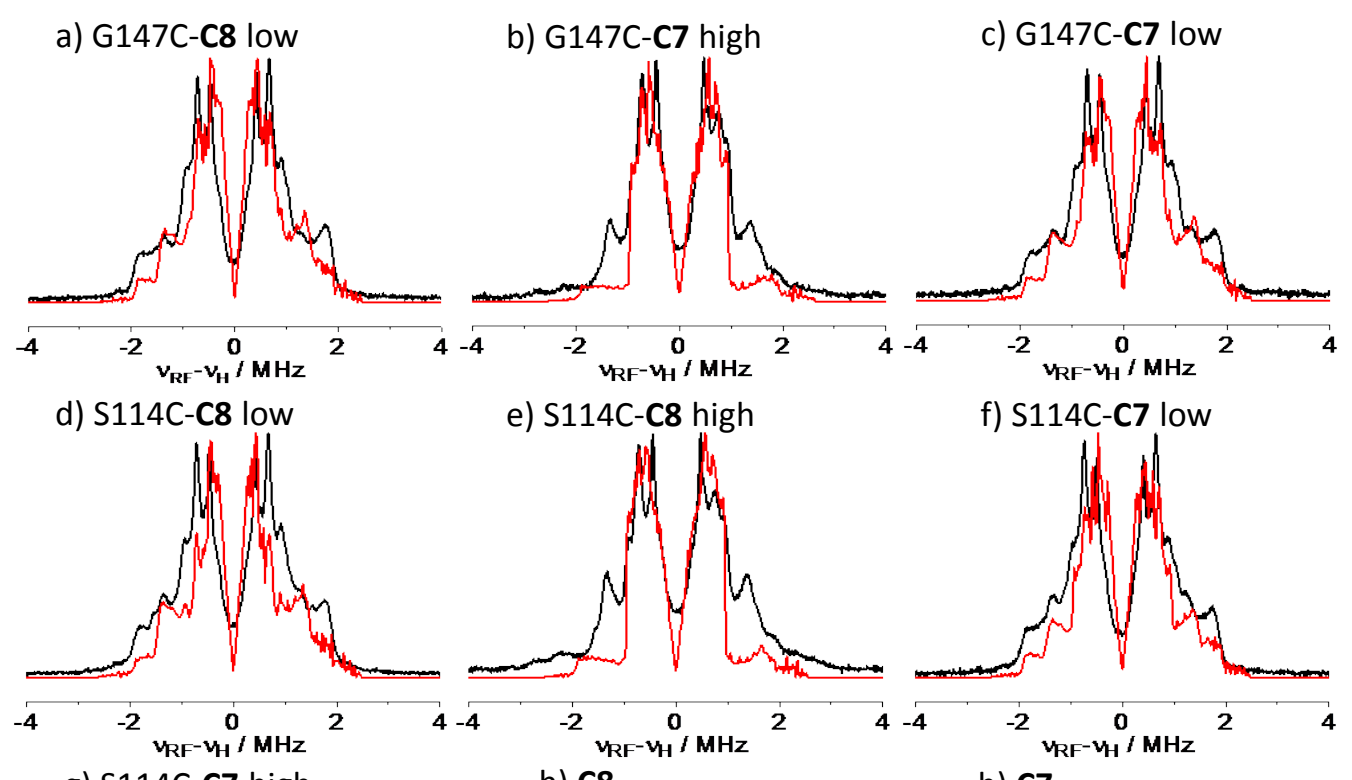

e) S114C-C8 high
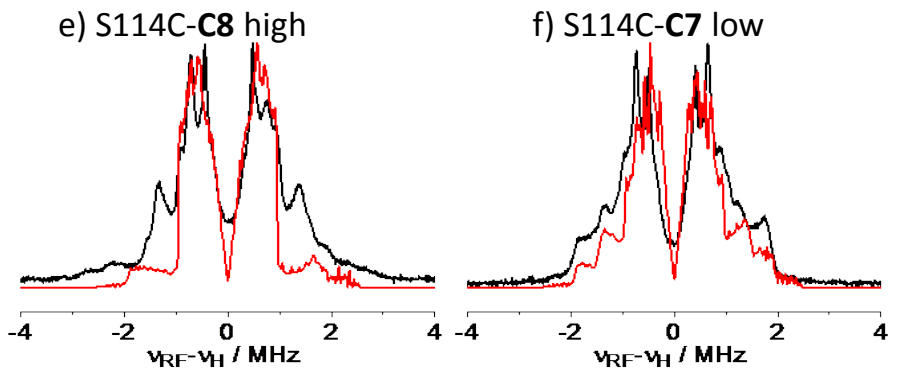

g) S114C-C7 high

h) $\mathbf{C 8}$

h) $\mathbf{C 7}$
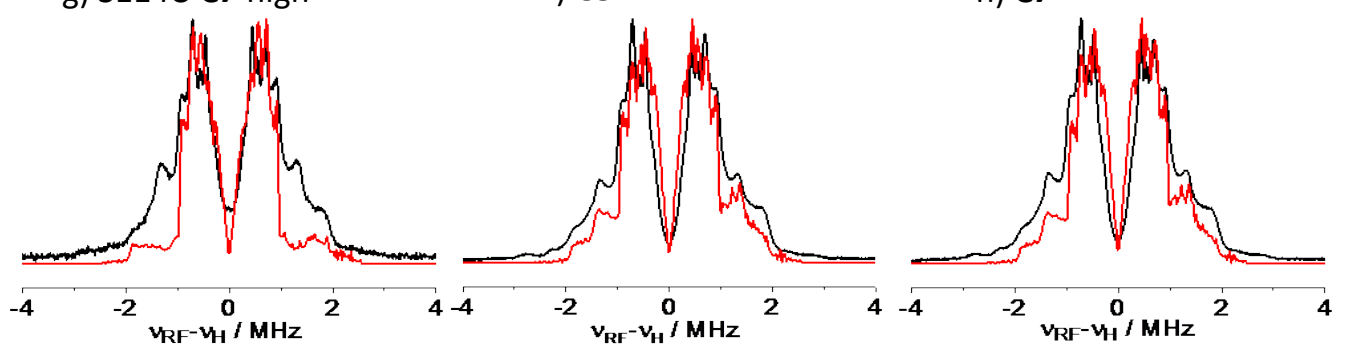

Figure S16. Same as Figure S15 but for $\beta=90^{\circ}$ for the class B protons. 


\section{DEER data and validations}
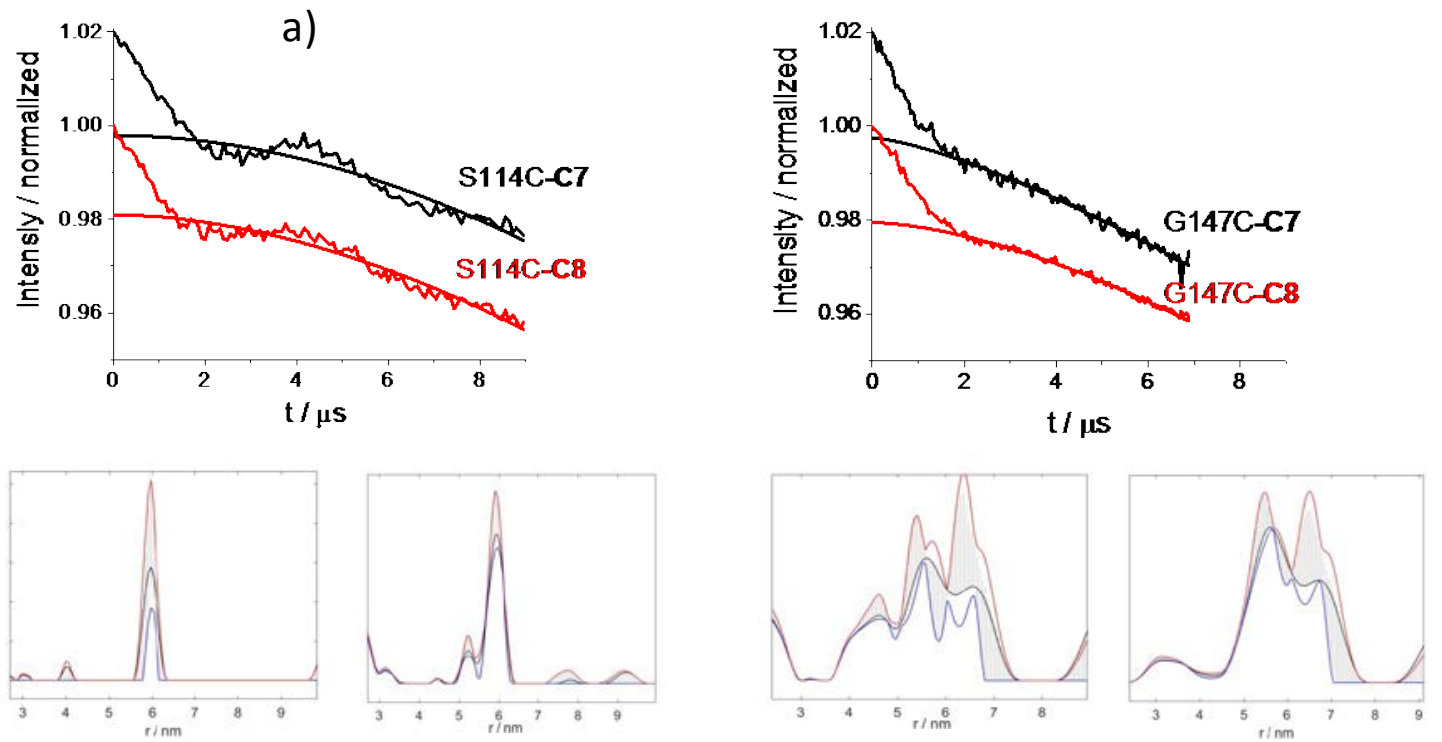

Figure S17. Primary DEER traces of the ERp29 mutants S114C (a) and G147C (b) labeled with C7 and C8 with the background correction function. The bottom traces show the uncertainty regions of the distance distributions as determined using DeerAnalysis 2015.
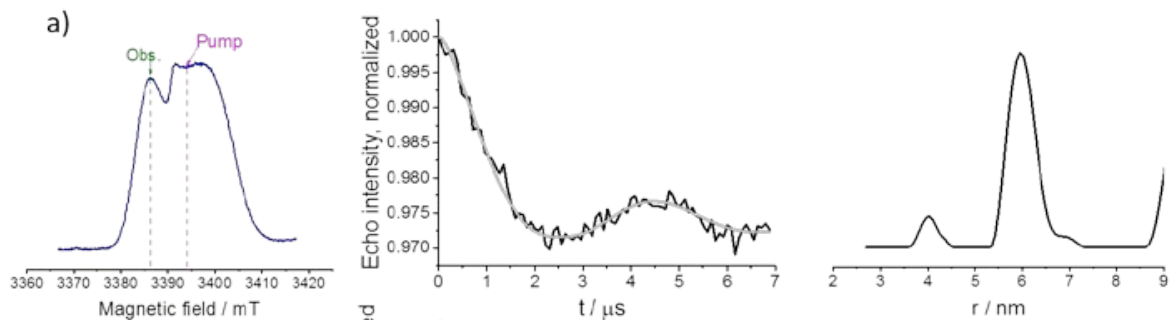

b)
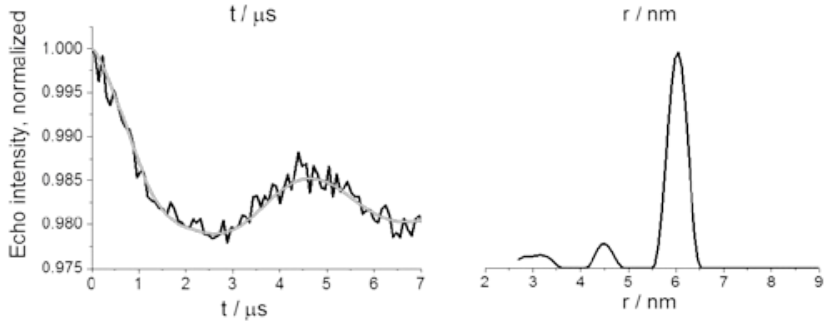

Figure S18. DEER results for S114C-C8 measured with dual and single mode cavities. (a) Left: ED-EPR spectrum showing the positions of the pump and observe pulses used for DEER measurements with a dual-mode cavity. Middle: Background-subtracted DEER trace measured with fitted trace (grey). Right: Corresponding distance distribution. (b) DEER results for S114C-C8, pH = 8, measured with a single mode cavity. Middle: Background-subtracted DEER trace with fitted trace (grey). Right: Corresponding distance distribution. 


\section{Simulations of ED-EPR spectra of other tags}

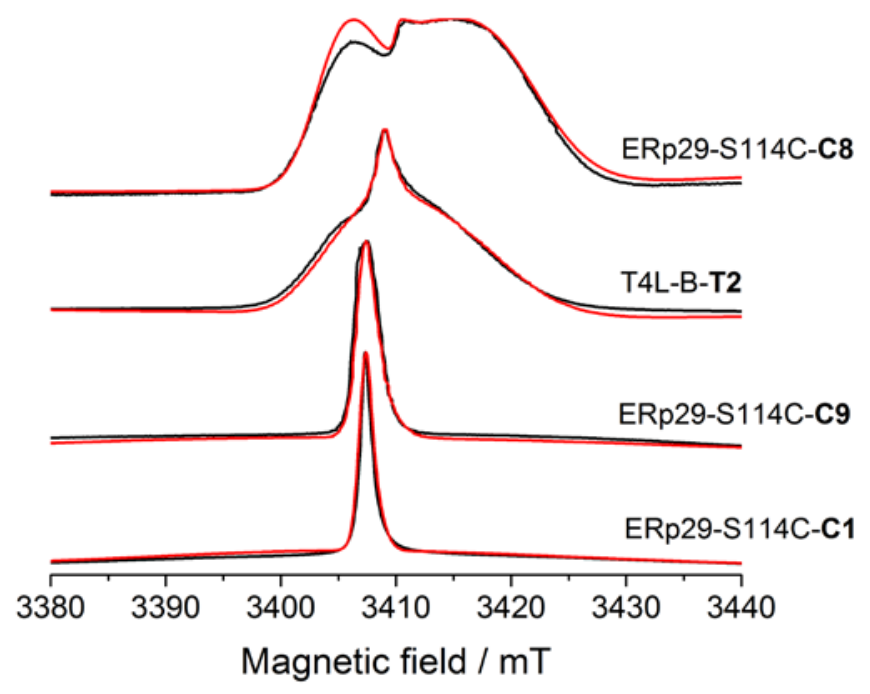

Figure S19. Simulations of the spectra shown in Figure 8 of the main text with the parameters listed in Table 3.

\section{References}

1. C.-H. Huang, J. Hammell, S. J. Ratnakar, A. D. Sherry and J. R. Morrow, Activation of a PARACEST agent for MRI through selective outersphere interactions with phosphate diesters. Inorg. Chem. 2010, 49, 5963-5970. 